\title{
Identification of a circRNA-miRNA-mRNA regulatory network for exploring novel therapeutic options for glioma
}

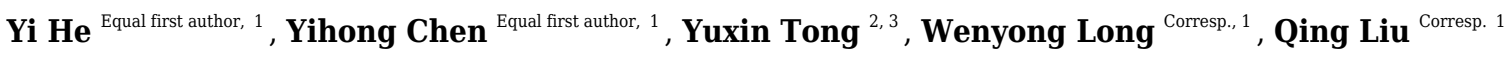 \\ ${ }^{1}$ Neurosurgery Department, Xiangya hospital Central South University, Changsha, Hunan, China \\ 3 Hunan Clinical Research Center of Ophthalmic Disease, Changsha, Hunan, China \\ Corresponding Authors: Wenyong Long, Qing Liu \\ Email address: wylongdr@csu.edu.cn, liuqingdr@csu.edu.cn
}

Background: Glioma is the most common brain neoplasm with a poor prognosis. Circular RNA (circRNA) and their associated competing endogenous RNA (ceRNA) network play critical roles in the pathogenesis of glioma. However, the alteration of the circRNA-miRNA-mRNA regulatory network and its correlation with glioma therapy haven't been systematically analyzed.

Methods: With GEO, GEPIA2, circBank, CSCD, Circlnteractome, mirWalk 2.0, and mirDIP 4.1, we constructed a circRNA-miRNA-mRNA network in glioma. LASSO regression and multivariate Cox regression analysis established a hub mRNA signature to assess the prognosis. GSVA was used to estimate the immune infiltration level. Potential anti-glioma drugs were forecasted using the cMap database and evaluated with GSEA using GEO data.

Results: A ceRNA network of 7 circRNAs (hsa_circ_0030788/0034182/0000227/0018086/0000229/0036592/0002765), 15 miRNAs(hsamiR-1200/1205/1248/1303/3925-5p/5693/581/586/599/607/640/647/6867-5p/767-3p/935), and 46 mRNAs (including 11 hub genes of ARHGAP11A, DRP2, HNRNPA3, IGFBP5, IP6K2, KLF10, KPNA4, NRP2, PAIP1, RCN1, and SEMA5A) was constructed. Functional enrichment showed they influenced majority of the hallmarks of tumors. Eleven hub genes were proven to be decent prognostic signatures for glioma in both TCGA and CGGA datasets. 46 LASSO regression significant genes were closely related to immune infiltration. Finally, five compounds (fulvestrant, tanespimycin, mifepristone, tretinoin, and harman) were predicted as potential treatments for glioma. Among them, mifepristone and tretinoin were proven to inhibit the cell cycle and DNA repair in glioma.

Conclusion: This study highlights the potential pathogenesis of the circRNA-miRNA-mRNA regulatory network and identifies novel therapeutic options for glioma. 
1 Identification of a circRNA-miRNA-mRNA regulatory

2 network for exploring novel therapeutic options for

3 glioma

4

Yi He ${ }^{1 \dagger}$, Yihong Chen ${ }^{1 \dagger}$, Yuxin Tong ${ }^{2,3}$, Wenyong Long ${ }^{1 *}$, Qing Liu ${ }^{1 *}$

${ }^{1}$ Neurosurgery Department, Xiangya Hospital, Central South University, Changsha, Hunan,

8 China

9 2Department of Ophthalmology of Second Xiangya Hospital, Central South University,

10 Changsha, China

${ }^{3}$ Hunan Clinical Research Center of Ophthalmic Disease, Changsha, China

These authors contributed equally to this work.

Corresponding Author:

Wenyong Long

Email: wylongdr@csu.edu.cn

Phone: +86-0731-89753035

Mail address: Xiangya Hospital, Central South University, Changsha, Hunan, China.

Qing Liu

20

Email: liuqingdr@csu.edu.cn

21

Phone: +86-0731-89753035

Mail address: Xiangya Hospital, Central South University, Changsha, Hunan, China.

\section{Abstract}

25

26

27

28

29

30

31

32

33

34

35

36

37

Background: Glioma is the most common brain neoplasm with a poor prognosis. Circular RNA (circRNA) and their associated competing endogenous RNA (ceRNA) network play critical roles in the pathogenesis of glioma. However, the alteration of the circRNA-miRNA-mRNA regulatory network and its correlation with glioma therapy haven't been systematically analyzed. Methods: With GEO, GEPIA2, circBank, CSCD, CircInteractome, mirWalk 2.0, and mirDIP 4.1, we constructed a circRNA-miRNA-mRNA network in glioma. LASSO regression and multivariate Cox regression analysis established a hub mRNA signature to assess the prognosis. GSVA was used to estimate the immune infiltration level. Potential anti-glioma drugs were forecasted using the cMap database and evaluated with GSEA using GEO data.

Results: A ceRNA network of 7 circRNAs (hsa_circ_0030788/0034182/0000227/0018086/0000229/0036592/0002765), 15 miRNAs(hsamiR-1200/1205/1248/1303/3925-5p/5693/581/586/599/607/640/647/6867-5p/767-3p/935), and 46 mRNAs (including 11 hub genes of ARHGAP11A, DRP2, HNRNPA3, IGFBP5, IP6K2, KLF10, KPNA4, NRP2, PAIP1, RCN1, and SEMA5A) was constructed. Functional enrichment showed they influenced majority of the hallmarks of tumors. Eleven hub genes were proven to be 
40 decent prognostic signatures for glioma in both TCGA and CGGA datasets. 46 LASSO

41 regression significant genes were closely related to immune infiltration. Finally, five compounds

42 (fulvestrant, tanespimycin, mifepristone, tretinoin, and harman) were predicted as potential

43 treatments for glioma. Among them, mifepristone and tretinoin were proven to inhibit the cell

44 cycle and DNA repair in glioma.

45 Conclusion: This study highlights the potential pathogenesis of the circRNA-miRNA-mRNA

46 regulatory network and identifies novel therapeutic options for glioma.

47

48

49

50

51

52

53

54

55

56

57

58

59

60

61

62

63

64

65

66

67

68

69

70

71

72

73

74

75

76

77

78

79

Keywords: glioma; circRNA; ceRNA network; novel therapy; biomarker

\section{Introduction}

Gliomas comprise majority of primary intracranial neoplasms with high heterogeneity and aggressiveness, resulting in a poor prognosis even after current standard combination treatments (Ostrom et al. 2018b; Wesseling \& Capper 2018). The five-year survival rate is only approximately 5\% (Ostrom et al. 2018a; Ostrom et al. 2018b; Wesseling \& Capper 2018). Recent advances in precision medicine, genomics, immunology, and other disciplines have uncovered multiple experimental therapies, such as targeted therapy, gene therapy, immunotherapy, and novel drug-delivery technologies that could possibly shed light on the treatment strategies for gliomas (Lapointe et al. 2018). Therefore, it is of great importance to explore the internal mechanisms of gliomas to identify new therapeutic targets.

Circular RNA (circRNA) is a type of non-coding RNA derived from the exon or intron region of a gene (Kristensen et al. 2019). Since there is no 5-3 polarity and polyA tail, circRNAs are more stable than linear RNAs (Kristensen et al. 2019; Meng et al. 2017). CircRNA regulates the expression of a series of genes by modulating every stage of mRNA metabolism, including sequestration of microRNAs (miRNA) or proteins, modulation of transcription, interference with splicing, and translation to produce polypeptides (Chen 2020; Wu et al. 2020). During the sequestration of miRNAs, circRNAs act as molecular sponges for miRNAs through their miRNA response elements (MREs), thereby de-repressing all target genes of the respective miRNA family (Hansen et al. 2013). Recently, circRNAs have been found to participate in multiple tumor phenotypes, including proliferation, invasion, metabolism, and immune response, making them promising diagnostic and prognostic markers as well as therapeutic targets for cancers (Chen 2020).

Recent studies have shown that instead of pure malignant cells, the core tumors are actually surrounded by a complex microenvironment, including the immune cells (Binnewies et al. 2018). Immune cells infiltrating the tumor microenvironment have been confirmed with the ability to predict the patients' clinical outcomes and also the efficacy of immunotherapy. Therefore, identifying immune cells infiltration, especially their pattern correlated with specific genes and gene signatures, is of great significance for estimation of the prognosis of GBM patients and the value of various therapies (Ali et al. 2016; Quail \& Joyce 2013). 
80 Here, by utilizing bioinformatics methods, we identified differentially expressed circRNAs 81 (DECs) in gliomas and studied their functions in gliomas as competing endogenous RNAs 82 (ceRNAs). The workflow diagram is shown in Fig. 1, where DECs were first acquired from the 83 circRNA-related microarray datasets of gliomas in the GEO database. Then, we forecasted and 84 collected their related miRNAs and their corresponding target genes and built a circRNA85 miRNA-mRNA regulatory network. Functional enrichment analyses were performed to 86 determine their potential roles in the pathogenesis of gliomas. Furthermore, the hub genes were 87 obtained through LASSO and multivariate Cox regression analyses and evaluated with ROC 88 curve analysis and K-M curve analysis. Subsequently, GSVA analysis was performed to 89 90 91 92

93

94 95 96 97 98 99

100

101

102

103

104

105

106

107

108

109

110

111

112

113

114

115

116

117

118

119 determine their correlation with immune infiltration. Finally, a connectivity map (CMap) was used to predict corresponding bioactive compounds and potential drugs for treatment, which were further assessed with GSEA in GEO database.

\section{Materials \& Methods}

\section{Data obtained and DECs acquired}

Microarray circRNA expression profile data of gliomas and corresponding normal tissues were screened and acquired from the Gene Expression Omnibus (GEO, https://www.ncbi.nlm.nih.gov/geo/) database, which is a public functional genomic database that allows users to query, locate, review, as well as download research and gene expression profiles (Barrett et al. 2013). DECs in the GSE109569 and GSE146463 datasets were analyzed and identified using GEO2R with the criteria of $\mid \log 2$ (fold change) $\mid>1$ and $P$ value $<0.05$. The circRNAs upregulated or downregulated in both datasets were selected for further analysis.

\section{Prediction of MREs}

We employed three public databases to predict the MREs of the selected DECs: CircBank (http://www.circbank.cn/index.html) is a comprehensive database with more than 140,000 human-annotated circRNAs from different sources, providing abundant information on circRNAs, including their predicted binding miRNAs (Liu et al. 2019). The cancer-specific circRNA database (CSCD, http://gb.whu.edu.cn/CSCD/) is a cancer-specific circRNA database incorporating more than 272,000 cancer-specific circRNAs, with the aim of predicting MRE sites, RNA binding protein (RBP) sites, and open reading frames (ORFs) for each circRNA (Xia et al. 2018). Circular RNA Interactome (CircInteractome, http://circinteractome.nia.nih.gov) is a web tool for mapping RBP and MRE sites on human circRNAs by searching public databases of circRNA, miRNA, and RBP. It has multiple functions, including identifying potential circRNAs that can act as RBP sponges (Dudekula et al. 2016). An overlap in at least two databases was the basis for considering candidate target miRNAs of these DECs used for further mRNA prediction. The regulatory roles of these miRNAs and related regulation pathways were assessed using DIANA-miRPath v3.0 (https://www.microrna.gr/miRPathv3), which is a powerful online software for functional analysis of miRNAs (Vlachos et al. 2015).

\section{Forecasting miRNA-mRNA interactions}


120

121

122

123

124

125

126

127

128

129

130

131

132

133

134

135

136

137

138

139

140

141

142

143

144

145

146

147

148

149

150

151

152

153

154

155

156

157

158

159

miRNA-mRNA interactions were predicted using two integrated miRNA databases. MiRWalk 2.0 (http://zmf.umm.uni-heidelberg.de/mirwalk2) is a web tool that provides information about validated or putative miRNA-mRNA interactions. For its prediction, 12 algorithms (miRWalk, Microt4, mirbridge Targetscan, RNAhybrid, RNA22, PITA, Pictar2, miRNAMap, miRDB, miRanda, and miRMap) were employed to ensure robustness (Dweep \& Gretz 2015). Here, targeted genes forecasted by at least seven algorithms, along with the validated genes, were selected as candidate genes from miRWalk 2.0. Meanwhile, mirDIP v4.1

(http://ophid.utoronto.ca/mirDIP/) is a miRNA database integrated across 30 different resources, capable of providing nearly 152 million human microRNA-target predictions (Tokar et al. 2018). In the mirDIP v4.1 database, genes predicted by at least 11 algorithms under the very high score class were selected as candidate genes from mirDIP v4.1.

\section{Obtaining DEGs and overlapped target genes}

The Gene Expression Profiling Interactive Analysis (GEPIA) web server is a valuable resource for gene expression analysis based on tumor and normal samples from the TCGA and GTEx databases. GEPIA2 is an updated and enhanced version with higher resolution and more functionalities (Tang et al. 2019). Through GEPIA2, We identified differentially expressed genes (DEGs) between glioblastoma (GBM) and normal tissues using the criteria of $\mid \log 2$ (fold change) $\mid>1$ and $P$ value $<0.01$. These DEGs were intersected with the candidate gene sets from miRWalk 2.0 and mirDIP v4.1. Overlapped mRNAs showing up in all three sets were taken as final target mRNAs and used for further analysis.

\section{Functional enrichment analysis of overlapped genes}

The Search Tool for the Retrieval of Interacting Genes database (STRING) is a database aimed at achieving a comprehensive and objective global network, including direct (physical) and indirect (functional) interactions (Szklarczyk et al. 2019). It was utilized to perform Gene Ontology (GO) analysis and Kyoto Encyclopedia of Genes and Genomes (KEGG) pathway enrichment analysis for the overlapped mRNA, with a setting $P<0.05$ and counts $>5$.

\section{Identification and assessment of hub genes}

Using mRNA expression profiles and clinical information from TCGA

(https://www.cancer.gov/tcga), the overlapping genes were consecutively analyzed with LASSO regression and multivariate Cox regression analysis, and independent prognostic genes were identified as hub genes. The total risk score of each sample was calculated as the sum of the multiplication of the expression value and the correlation coefficient of each gene. Patients with higher $50 \%$ or lower $50 \%$ of risk score were defined as high-risk or low-risk groups respectively. Their value as a prognostic signature for gliomas, as well as their corresponding sensitivity and specificity, were evaluated using K-M curve analysis and ROC curve analysis both in the TCGA training dataset and the external CGGA validation dataset (http://www.cgga.org.cn/) (Zhao et al. 2021). Sample IDs of the CGGA samples used in this study was listed in supp_table4. The protein level expression differences of hub genes were further confirmed with immunohistochemistry (IHC) images from The Human Protein Atlas (HPA) database (Uhlen et al. 2015). 
160

161

162

163

164

165

166

167

168

169

170

171

172

173

174

175

176

177

178

179

180

181

182

183

184

185

186

187

188

189

190

191

192

193

194

195

196

197

198

199

\section{Construction of a circRNA-miRNA-mRNA network}

Cytoscape is an open-source software for the integration of molecular interaction network data and the establishment of powerful visualization (Shannon et al. 2003). Here, it was used to construct a circRNA-miRNA-mRNA regulatory network.

\section{Assessment of Immune Cell Infiltration}

Gene set variation analysis (GSVA) is a gene set enrichment method and an open source software package for $\mathrm{R}$, which can estimate the variation of pathway activity over a sample population in an unsupervised manner (Hanzelmann et al. 2013). To estimate the immune cell infiltration level, we applied single-sample gene-set enrichment analysis (ssGSEA), which is a built-in algorithm of the GSVA package, using the RNA-seq data and related clinical data from the TCGA-GBMLGG dataset. Gene expression features of 24 immune cells were acquired from a previous study (Bindea et al. 2013), and the correlation with immune cell infiltration was obtained for the genes that passed LASSO regression analysis. Sample IDs of the TCGAGBMLGG samples used in this study was listed in supp_table5.

\section{Connectivity Map (CMap) analysis and assessment}

The connectivity map (CMap) is a collection of genome-wide transcriptional expression data from human cell lines treated with various drugs or compounds. Functional connections between drugs, genes, and diseases were then uncovered using pattern-matching algorithms and features of common gene expression changes (Lamb 2007; Lamb et al. 2006). Using the hub genes from multivariate Cox regression analysis, candidate compounds with negative connectivity scores were identified as promising candidate therapeutic approaches. The available related RNA-seq data were acquired from GEO and used for GSEA analysis to explore the effects of those drugs on gliomas.

\section{Statistical Analysis}

R software (version 4.0.3) was used for all statistical analyses, and p-values $<0.05$ were considered statistically significant. The Glmnet package and survival package were utilized for LASSO regression and multivariate Cox regression analysis, respectively. GGally and rms packages were used to evaluate and remove the co-linearity between samples. K-M curve analysis was performed using the Survminer package. ROC curve analysis was performed using the survival ROC package. Visualization was achieved using the ggplot2, pheatmap, or plotROC packages.

\section{Results}

\section{Acquiring 8 DECs in gliomas}

To explore the potential function of circRNAs and the corresponding ceRNA network in glioma, DECs from GSE109569 (3 glioma samples vs. 3 normal samples) and GSE146463 (8 glioma samples vs. 3 normal samples) datasets were obtained using GEO2R from the GEO database. Genes with $P<0.05$ and a $\mid \log 2$ (fold change) $\mid>1$ were considered significant DECs. Through the intersection of two DEC datasets, six upregulated and two downregulated circRNAs were 
200 identified and chosen as research objects in this study. The differences in expression between 201 gliomas and normal tissues are shown in Fig. 2a-b. The basic features of these eight circRNAs 202 are listed in Table 1. The circRNAs' accurate expression values in the GEO datasets are 203 summarized in supp_table1.

204 Identification of circRNA-miRNA interactions

205 To explore the roles of these eight circRNAs as ceRNAs in glioma, three online databases, 206 namely circBank, CSCD, and CircInteractome, were utilized to collect potential target miRNAs. 207 Six out of eight circRNAs were recorded in the CSCD database, and their structures of MRE, 208 RBP, and ORF are shown in Fig. 2c. A total of 15 miRNAs and 18 circRNA-miRNA 209 interactions were identified by at least two databases, including hsa_circ_0030788-miR210 5693/miR-6867-5p/miR-607/miR-1248/miR-586/miR-599, hsa_circ_0034182-miR-1200, 211 hsa_circ_0000227-miR-647/miR-1303/miR-767-3p, hsa_circ_0018086-miR-1303/miR-3925212 5p/miR-581, hsa_circ_0000229-miR-935/miR-640, hsa_circ_0036592-miR-1205/miR-767-3p, 213 and hsa_circ_0002765- miR-587/miR-767-3p. The functions of these miRNAs in tumors 214 reported in PubMed were summarized in Table 2. Among them, miR-1303, miR-581, miR-586, 215 miR-599, miR-607, miR-647, miR-767-3p, miR-935, and miR-1248 have been extensively 216 reported to regulate the progression of various tumors as promoters or suppressors. DIANA217 miRPath was then used to probe signaling pathways involving 15 unique miRNAs. As shown in 218 Fig. 3a, these miRNAs are involved in multiple pathways of glioma, including the FoxO 219 signaling pathway, phosphatidylinositol signaling pathway, and TGF $\beta$ signaling pathway. 220 Furthermore, given that the expression levels of the miRNAs are critical for their biological 221 functions, we checked the expression levels of those 15 miRNAs in glioma tissues with CGGA 222 data and GEO data, as shown in (supp_table.2-3). Different data sets showed slightly different 223 224

225

226

227

228

229

230

231

232

233

234

235

236

237

238

239 expression of those miRNAs. And their expression values vary quite a lot from sample to sample. But in general, according to those two datasets, the miR607 and miR587 have relatively lower expression levels.

\section{Obtaining target mRNAs in the ceRNA network}

To forecast the target genes of these miRNAs, miRWalk 2.0, mirDIP v4.1, and GEPIA2 were utilized in this study. A total of $7688 \mathrm{mRNAs}$ were validated or predicted by at least seven algorithms in miRWalk 2.0. A total of 3370 genes were forecasted by more than 10 algorithms in mirDIP v4.1 to be targets of those miRNAs. In GEPIA2, 7652 genes were identified as significant DEGs with $\mathrm{P}<0.01$ and $\mid \log 2$ (fold change) $\mid>1$. The chromosomal distribution of DEGs is displayed in Fig. 3b. Thereafter, through the intersection of all three gene sets, we obtained a total of 1076 target mRNAs involved in the ceRNA network (Fig. 3c). whose overall expression levels were not significantly correlated with genders (Fig. S1).

\section{Function enrichment analyses}

To explore the potential functions of the 1076 target genes, GO and KEGG signal pathway enrichment analyses were performed using STRING. In terms of biological processes, these target genes covered majority of the hallmark pathways of tumors, including cell growth, cell 
240 cycle, proliferation, differentiation, migration, apoptosis, cell death, immune response,

241 angiogenesis, as well as some well-known cancer-related pathways such as the Wnt and TGF $\beta$

242 signaling pathways, which were also exhibited in KEGG enrichment results. In addition, KEGG

243 also showed enrichment in some other essential pathways, such as the cell cycle, p53, Hippo,

244 MAPK, and stemness regulating pathways. The compositions of the target genes encompassed a

245 wide range of cellular components and molecular functions from cytosol to synapse as well as

246 from DNA binding to enzyme binding. Visualization of the enrichment results is displayed in

247 Fig. 4. Taken together, functional enrichment results indicated that the ceRNA network is

248 extensively involved in the pathogenesis of gliomas.

249 Identification and assessment of hub genes

250 Utilizing gene expression data and corresponding clinical data from the TCGA-GBMLGG data

251 set, we applied LASSO regression analysis to the 1076 target genes and obtained 46 significant

252 candidate genes which were further used for multivariate Cox regression analysis (Fig. 5).

253 Eventually, 11 independent prognosis-related hub genes were identified, including

254 ARHGAP11A, DRP2, HNRNPA3, IGFBP5, IP6K2, KLF10, KPNA4, NRP2, PAIP1, RCN1,

255 and SEMA5A. Thereafter, we checked the human protein atlas database to confirm the protein

256 expression level changes of the 11 hub genes. Three of them (DRP2, IGFBP5, KLF10) are not

257 provided with protein expression information. Two (ARHGAP11A, NRP2) of them showed no

258 big difference between normal and glioma samples. The other six of them (HNRNPA3, IP6K2,

259 KPNA4, PAIP1, RCN1, SEMA5A) showed significantly increased protein expression, consistent

260 with our results (supp_fig2). The genes with supporting protein level results could be of more

261 importance for further future validation study. But given the limited sample size of this part of

262 data, we still took all those 11 genes as our subjects of analysis.

263 Based on the expression value and correlation coefficients, we integrated these 11 genes as a

264 whole signature and computed the total risk score of each sample to divide glioma patients into

265 high- and low-risk groups. The K-M curve analysis indicated that the overall survival of the

266 high-risk group was significantly shorter than that of the low-risk group (Fig. 6a). ROC curve

267 analysis further showed decent sensitivity and specificity of this signature in predicting the

268 prognosis of glioma patients (Fig. 6b). An external independent CCGA dataset was introduced to

269 validate the results of the K-M curve and ROC curve analyses, and similar conclusions were

270 obtained (Fig. 6c-d).

271 Construction of a circRNA-miRNA-mRNA network

272 To present the relationship between circRNA, miRNA, and mRNA, a ceRNA network consisting

273 of seven circRNAs, 15 miRNAs, and 46 mRNAs was constructed using Cytoscape as shown in

274 Fig. 7. The five upregulated circRNAs influenced nine miRNAs and 13 mRNAs, while the two

275 downregulated circRNAs targeted seven miRNAs and eight mRNAs. Meanwhile, 25 mRNAs

276 were under the two-way regulation of both groups of circRNAs.

277 Immune cell infiltration features of prognosis-related genes

278 Gene expression profiles from TCGA-GBMLGG dataset and immune cell signatures from a

279 previous study were used for immune infiltration analysis with the GSVA package (Bindea et al. 
280

281

282

283

284

285

286

287

288

289

290

291

292

293

294

295

296

297

298

299

300

301

302

303

304

305

306

307

308

309

310

311

312

313

314

315

316

317

318

319

2013; Hanzelmann et al. 2013). Spearman correlation between infiltration levels of 24 immune cells and 46 prognosis-related genes demonstrated that their expression levels were closely related to tumor microenvironment immune infiltration (Fig. 8).

\section{Candidate compounds from CMap and assessment}

Notably, in order to explore the practical value of this study, the candidate compounds that might have effects on gliomas were predicted by CMap with the hub genes we screened out (Table 3). Based on the enrichment correlation coefficient, drugs such as fulvestrant, tanespimycin, mifepristone, tretinoin, and Harman are the most promising potential therapeutic options for gliomas. With GSEA analysis on RNA-seq data from GEO (GSE59262 for mifepristone; GSE141789, GSE17227, and GSE61002 for tretinoin), mifepristone and tretinoin were proven to inhibit cell cycle and DNA repair pathways (Fig. 9). These novel therapeutic options would require more preclinical and clinical studies for further validation.

\section{Discussion}

CircRNA can act as a molecular sponge for miRNAs to de-repress all target genes of these miRNAs (Kristensen et al. 2019; Meng et al. 2017). Accumulating evidence has shown that this circRNA-miRNA-mRNA network plays an important role in the pathogenesis of gliomas, encompassing a wide range of phenotypes, such as proliferation, migration, and invasion (Chen et al. 2019; Ding et al. 2020; Wang et al. 2018). Therefore, circRNAs and miRNAs are increasingly regarded as promising therapeutic targets or diagnostic biomarkers. GBM can be divided into different subsets with diverse anticancer responses to various therapies as a group of heterogeneous intracranial neoplasms with distinct histopathological and molecular biological characteristics (Lapointe et al. 2018). Thus, there is an urgent need to establish reliable risk stratification methods to classify GBM patients into various risk groups to benefit from various treatment strategies. Many studies have explored the prognostic signatures of gliomas in the context of epigenetic modifications or lncRNAs (Niu et al. 2020; Pan et al. 2021). However, so far, there has been no comprehensive and in-depth study of the molecular signatures of circRNArelated ceRNA networks in gliomas. Therefore, in this study, we constructed a circRNAmiRNA-mRNA network in glioma to help understand its pathogenesis as well as aid in risk stratification and therapeutic decision-making.

In this study, eight DECs (hsa_circ_0001156, hsa_circ_0030788, hsa_circ_0034182, hsa_circ_0000227, hsa_circ_0018086, hsa_circ_0000229, hsa_circ_0036592, and hsa_circ_0002765) were identified as DECs in the first step. To the best of our knowledge, all of them were found to be abnormally expressed in glioma for the first time and have not been studied so far, which makes them potential novel biomarkers or therapeutic targets.

Seven out of eight circRNAs (except for hsa_circ_0001156) were identified as ceRNAs to bind 15 miRNAs (hsa-miR-1200, hsa-miR-1205, hsa-miR-1248, hsa-miR-1303, hsa-miR-3925-5p, hsa-miR-5693, hsa-miR-581, hsa-miR-586, hsa-miR-599, hsa-miR-607, hsa-miR-640, hsa-miR647, hsa-miR-6867-5p, hsa-miR-767-3p, and hsa-miR-935). As for hsa_circ_0001156, it might still be involved in the pathogenesis of gliomas through functions other than miRNA sponges,

Peer] reviewing PDF | (2021:05:61464:1:1:NEW 26 Jun 2021) 
320

321

322

323

324

325

326

327

328

329

330

331

332

333

334

335

336

337

338

339

340

341

342

343

344

345

346

347

348

349

350

351

352

353

354

355

356

357

358

359

such as coding proteins, interacting with RNA binding proteins, or modulating the stability of mRNAs.

As shown in Table 2, among the 15 miRNAs identified, miR-581, miR-586, and miR-1248 were reported to promote tumor progression, while miR-599, miR-607, and miR-767-3p were shown to be tumor suppressors in various cancers. Some studies have shown contradictory results regarding the effects of miR-1303, miR-647, and miR-935 on some tumors. This could partly result from differences in the cell lines used, the phenotypes selected, or the pathways studied. However, further research is needed to resolve these discrepancies. Nevertheless, it is noteworthy that miR-647, miR-767-3p, and miR-935 reportedly suppress the progression of glioma through multiple regulatory axes, including miR-647/HOXA9, miR-935/FZD6, miR935/HIF $1 \alpha$, or miR-767-3p itself, making them promising biomarkers and therapeutic targets for glioma. In addition, the other six miRNAs without previous studies on tumors (miR-1200, miR1205, miR-3925-5p, miR-5693, miR-640, and miR-6867-5p) could also be novel fields worth exploring, which could possibly lead to unexpected discoveries.

Given the correlation between miRNAs expression levels and their biological functions, we further checked their expression levels in glioma tissues with CGGA data and GEO datasets, as shown in (supp_table.2-3). According to those two datasets, the miR607 and miR587 have relatively lower expression levels, implying they could be less important in glioma studies. And miR581 has relatively higher expression, giving it higher importance for glioma. But it is noteworthy that different datasets showed considerably different expression of those miRNAs, probably due to the different platforms used and various samples chosen. And their expression values vary quite a lot from sample to sample even for the same dataset. So, their actual expression levels still await to be examined.

CircRNAs fulfill their functions by de-repressing the target genes of miRNAs. Therefore, to further explore the effects of circRNAs on glioma, 1076 overlapping target genes were collected and used for functional enrichment analyses. The ten major hallmarks of tumors include sustaining proliferative signaling, evading growth suppressors, resisting cell death, enabling replicative immortality, inducing angiogenesis, activating invasion and metastasis, reprogramming of energy metabolism, evading immune destruction, genome instability and mutation, and dysregulating cellular energetics (Hanahan \& Weinberg 2011), most of which were covered in our enrichment result of biological processes. This indicates that the ceRNA network we built here is extensively involved in the initiation and progression of gliomas. The pathway enrichment results uncovered the involvement of many essential tumor-related pathways such as the Wnt, TGF $\beta$, cell cycle, p53, Hippo, MAPK, and stemness regulating pathways. Wnt signaling, commonly divided into $\beta$-catenin-dependent (canonical) and independent (non-canonical) signaling, is one of the key cascades regulating development (Klaus \& Birchmeier 2008). Its role in carcinogenesis has mostly been described in colorectal cancer, along with some other cancer entities (Zhan et al. 2017). The transforming growth factor (TGF)$\beta$ signaling pathway is deregulated in many diseases and has dual functions in cancers. It suppresses tumors in healthy cells and early stage cancer cells but promotes tumorigenesis, 
360

361

362

363

364

365

366

367

368

369

370

371

372

373

374

375

376

377

378

379

380

381

382

383

384

385

386

387

388

389

390

391

392

393

394

395

396

397

398

399

metastasis, and chemoresistance in late-stage cancer (Colak \& Ten Dijke 2017). p53 is a tumor suppressor protein that regulates cell growth by promoting apoptosis and DNA repair under stressful conditions (Kanapathipillai 2018). The Hippo pathway largely consists of a kinase cascade (MST1/2 and LATS1/2) and downstream transcriptional coactivators (YAP and TAZ), controlling transcriptional programs involved in cell proliferation, survival, mobility, stemness, and differentiation (Ma et al. 2019). The MAPK/ERK pathway is a chain of proteins that communicate signals from a receptor on the cell surface to the DNA in the nucleus of the cell (Orton et al. 2005). Alteration of this pathway is often a necessary step in the development of many cancers (Drosten \& Barbacid 2020). Cancer stem cells are capable of sustaining tumors by aiding metastasis, therapy resistance, and tumor microenvironment maintenance, making the stemness regulation key traits and mechanisms for tumor progression (Saygin et al. 2019). All of these pathways, which were under the control of the ceRNA network we constructed in this study, have been shown to participate in the initiation or progression of gliomas (He et al. 2019; Lan et al. 2019; Lee et al. 2017; Ma et al. 2018; Masliantsev et al. 2021; Zhao et al. 2019). Altogether, these 1076 target genes, regulated indirectly by the circRNAs identified in the present study, play essential roles in the pathogenesis of gliomas.

Thereafter, LASSO regression analysis and multivariate Cox regression analysis were applied to the 1076 genes consecutively. Forty-six LASSO significant genes and 11 independent prognosisrelated hub genes were identified (ARHGAP11A, DRP2, HNRNPA3, IGFBP5, IP6K2, KLF10, KPNA4, NRP2, PAIP1, RCN1, and SEMA5A). Among them, ARHGAP11A, DRP2, HNRNPA3, KLF10, PAIP1, and RCN1 have not yet been studied in gliomas. Three mRNAs were identified as oncogenes in gliomas, which was consistent with our multivariate Cox regression analysis result: IGFBP5 can increase cell invasion and inhibit cell proliferation via the EMT and Akt signaling pathways in GBM (Dong et al. 2020); IP6K2 was reported to promote cell proliferation and inhibit cell apoptosis under the regulation of the LINC00467/miR-339-3p axis (Liang \& Tang 2020); and KPNA4 is capable of facilitating epithelial-mesenchymal transition in glioma, which can be suppressed by miR-181b, a tumor-suppressive miRNA (Wang et al. 2015). Surprisingly, the roles of the other two mRNAs in glioma were shown to be different from our analysis result: NRP2 promoted glioma cell growth, invasion, and angiogenesis (Zheng et al. 2013); and SEMA5A, whose expression is markedly reduced in higher grades of glioma, can impede motility and promote differentiation of human gliomas ( $\mathrm{Li}$ \& Lee 2010). This discrepancy might result from the fact that we used survival data for analysis, and those genes were studied only in vitro for some specific phenotypes, while the in vivo result of survival might be influenced by multiple other conditions and phenotypes (such as immune response and therapy sensitivity). However, the functions of these genes require further experimental verification. Nevertheless, the K-M curve analysis and ROC curve analysis in both training and external validation datasets proved that these genes together are a competent signature for predicting the prognosis of gliomas.

In addition, we checked the human protein atlas database to confirm the protein expression level changes of the 11 hub genes. Three of them (DRP2, IGFBP5, KLF10) are not provided with 
400 protein expression information. Two (ARHGAP11A, NRP2) of them showed no big difference 401 between normal and glioma samples. The other six of them (HNRNPA3, IP6K2, KPNA4, 402 PAIP1, RCN1, SEMA5A) showed significantly increased protein expression, consistent with our 403 results (supp_fig2). This doesn't mean that DRP2, IGFBP5, KLF10, ARHGAP11A and NRP2 404 are not essential genes for glioma pathogenesis, given the missing data and limited sample size 405 we found. But still this could give us some hints that those genes with supporting HPA results 406 (namely HNRNPA3, IP6K2, KPNA4, PAIP1, RCN1, SEMA5A) could be more promising 407 targets for future further validation studies.

408 Recently, circRNAs were reported to play a significant role in multiple immune-related 409 biological processes, including innate and adaptive immune responses, immune cell homeostasis, 410 immune recognition, and anti-tumor immunity (Yan \& Chen 2020; Zhang et al. 2020).

411 Comprehensive recognition of circRNA-mediated immune cell infiltration in glioma can provide 412 novel insights into risk stratification and clinical therapeutic strategies. Hence, we profiled tumor 413 microenvironment immune cell infiltration utilizing 46 prognosis-related genes from the LASSO 414 regression analysis as shown in Fig. 8. The current consensus is that the anti-tumor immune 415 response in glioma is largely suppressed by brain-resident microglial cells and bone marrow416 derived macrophages, and is mainly promoted by CD8+ T cells (Pinton et al. 2019). However, 417 the roles of other immune cells, such as B cells, are still debatable (Pinton et al. 2019; von 418 Roemeling et al. 2020). Our results showed that generally the genes that were positively related 419 to macrophages were negatively correlated with CD8+ T cells, and vice versa. This indicated that 420 these genes could play considerable roles in immune infiltration switch of the tumor 421 microenvironment. However, it should be noted that there is still a lack of systemic immune cell 422 markers for gliomas. Existing immune markers are mostly constructed in other tumors, and some 423 glioma-specific immune cells (such as microglial cells) still lack convincing specific markers.

424 Specific immune cell markers for gliomas are urgently needed for a robust assessment of 425 immune infiltration and understanding of immune response mechanisms in gliomas.

426 Glioma is one of the most drug-resistant malignancies with frequent recurrence after 427 chemotherapy, making it necessary to explore novel compounds or drugs that may have a 428 therapeutic effect. Here, with the hub genes identified, some potential drugs were acquired from 429 the CMap database. Although the prediction of CMap was mostly based on experiments in 430 prostate cancer and leukemia cell lines, the effects of these drugs have also been verified in 431 multiple other tumors. For instance, fulvestrant is a selective estrogen receptor degrader that has 432 been extensively studied for its therapeutic effects in breast cancer (Slamon et al. 2020). 433 Harmane is a tremorigenic $\beta$-carboline capable of inhibiting mitochondrial viability and 434 increasing reactive oxygen species levels (Khan et al. 2017). Its semi-synthetic derivative, B-9-3, 435 showed an anti-proliferative effect in lung cancer, breast cancer, and colorectal carcinoma cell 436 lines via induction of apoptosis and inhibition of cell migration (Daoud et al. 2014). Some of 437 these drugs have been proven to interfere with the progression of gliomas. Tanespimycin is a 438 well-characterized HSP90 inhibitor that can inhibit the growth of GBM and synergize with 439 radiation (Sauvageot et al. 2009). Mifepristone was reported to be a potential therapy for 
440 reducing angiogenesis and TMZ resistance in GBM (Llaguno-Munive et al. 2020). Tretinoin, an

441 all-trans retinoic acid, was shown to significantly induce apoptosis and suppress stemness in

442 GBM (Chen et al. 2014; Hu et al. 2017). Importantly, mifepristone and tretinoin were shown to

443 inhibit cell cycle and DNA repair of glioma according to our own GSEA analysis result (Fig. 9).

444 Given that radiation and temozolomide, the major non-surgical treatments for glioma, both work

445 through inducing DNA damage, those novel drugs could be promising supplementary therapeutic

446 treatments which can be applied in combination with radiotherapy or chemotherapy.

447 Several limitations of this study should be considered. The construction of

448 circRNA/miRNA/mRNA regulatory networks and the prediction of therapeutic drugs largely

449 relied on a series of bioinformatics algorithms and databases, whose authenticity and accuracy

450 still await the verification of numerous experiments. Therefore, we adopted and integrated

451 multiple databases for all predictions in the present study to improve robustness. In addition, the

452 retrospective research design could display some statistical bias and the traditional bulk sequence

453 transcriptome data would lack comprehensive exploration of intra-tumoral heterogeneity. A

454 prospective study design and utilization of single-cell omics techniques will help address this

455 issue and provide more accurate and reliable results in the future. However, based on

$456 \mathrm{circRNA} / \mathrm{miRNA} / \mathrm{mRNA}$ regulatory networks, we established a superior predictive signature to

457 assess the clinical outcomes of patients with GBM and forecasted some promising candidate

458 drugs.

459

460

461

462

463

464

465

466

467

468

469

470

471

\title{
Conclusions
}

Through the construction of a circRNA/miRNA/mRNA regulatory network in glioma and the combination of survival analysis, this study successfully identified 11 circRNA-related mRNA signatures to predict the prognosis of GBM patients. Additionally, we determined that circRNAregulated hub genes were correlated with specific immune cell infiltration levels and proposed some potential therapeutic options. Comprehensively exploring the circRNA/miRNA/mRNA regulatory network in GBM will enhance our understanding of the pathogenesis and immune infiltration features of glioma, promote treatment strategies, and improve clinical outcomes.

\section{Acknowledgements}

All contributors to this study are included in the list of authors.

472

473

474

475

476

477

478

479

480

\section{References}

\begin{abstract}
Ali HR, Chlon L, Pharoah PD, Markowetz F, and Caldas C. 2016. Patterns of Immune Infiltration in Breast Cancer and Their Clinical Implications: A Gene-Expression-Based Retrospective Study. PLoS Med 13:e1002194. 10.1371/journal.pmed.1002194

Barrett T, Wilhite SE, Ledoux P, Evangelista C, Kim IF, Tomashevsky M, Marshall KA, Phillippy $\mathrm{KH}$, Sherman PM, Holko M, Yefanov A, Lee H, Zhang N, Robertson CL, Serova N, Davis S, and Soboleva A. 2013. NCBI GEO: archive for functional genomics data sets-update. Nucleic Acids Res 41:D991-995. 10.1093/nar/gks1193
\end{abstract}


481

482

483

484

485

486

487

488

489

490

491

492

493

494

495

496

497

498

499

500

501

502

503

504

505

506

507

508

509

510

511

512

513

514

515

516

517

518

519

520

521

522

523

524

525

526

527

528

529

530

531

Bindea G, Mlecnik B, Tosolini M, Kirilovsky A, Waldner M, Obenauf AC, Angell H, Fredriksen T, Lafontaine L, Berger A, Bruneval P, Fridman WH, Becker C, Pages F, Speicher MR, Trajanoski Z, and Galon J. 2013. Spatiotemporal dynamics of intratumoral immune cells reveal the immune landscape in human cancer. Immunity 39:782-795. 10.1016/j.immuni.2013.10.003

Binnewies M, Roberts EW, Kersten K, Chan V, Fearon DF, Merad M, Coussens LM, Gabrilovich DI, Ostrand-Rosenberg S, Hedrick CC, Vonderheide RH, Pittet MJ, Jain RK, Zou W, Howcroft TK, Woodhouse EC, Weinberg RA, and Krummel MF. 2018. Understanding the tumor immune microenvironment (TIME) for effective therapy. Nat Med 24:541-550. 10.1038/s41591-018-0014-X

Chen J, Chen T, Zhu Y, Li Y, Zhang Y, Wang Y, Li X, Xie X, Wang J, Huang M, Sun X, and Ke Y. 2019. circPTN sponges miR-145-5p/miR-330-5p to promote proliferation and stemness in glioma. J Exp Clin Cancer Res 38:398. 10.1186/s13046-019-1376-8

Chen LL. 2020. The expanding regulatory mechanisms and cellular functions of circular RNAs. Nat Rev Mol Cell Biol 21:475-490. 10.1038/s41580-020-0243-y

Chen PH, Shih CM, Chang WC, Cheng CH, Lin CW, Ho KH, Su PC, and Chen KC. 2014. MicroRNA-302b-inhibited E2F3 transcription factor is related to all trans retinoic acidinduced glioma cell apoptosis. J Neurochem 131:731-742. 10.1111/jnc.12820

Colak S, and Ten Dijke P. 2017. Targeting TGF-beta Signaling in Cancer. Trends Cancer 3:5671. 10.1016/j.trecan.2016.11.008

Daoud A, Song J, Xiao F, and Shang J. 2014. B-9-3, a novel beta-carboline derivative exhibits anti-cancer activity via induction of apoptosis and inhibition of cell migration in vitro. Eur J Pharmacol 724:219-230. 10.1016/j.ejphar.2013.12.038

Ding C, Yi X, Wu X, Bu X, Wang D, Wu Z, Zhang G, Gu J, and Kang D. 2020. Exosomemediated transfer of circRNA CircNFIX enhances temozolomide resistance in glioma. Cancer Lett 479:1-12. 10.1016/j.canlet.2020.03.002

Dong C, Zhang J, Fang S, and Liu F. 2020. IGFBP5 increases cell invasion and inhibits cell proliferation by EMT and Akt signaling pathway in Glioblastoma multiforme cells. Cell Div 15:4. 10.1186/s13008-020-00061-6

Drosten M, and Barbacid M. 2020. Targeting the MAPK Pathway in KRAS-Driven Tumors. Cancer Cell 37:543-550. 10.1016/j.ccell.2020.03.013

Dudekula DB, Panda AC, Grammatikakis I, De S, Abdelmohsen K, and Gorospe M. 2016. Circlnteractome: A web tool for exploring circular RNAs and their interacting proteins and microRNAs. RNA Biol 13:34-42. 10.1080/15476286.2015.1128065

Dweep H, and Gretz N. 2015. miRWalk2.0: a comprehensive atlas of microRNA-target interactions. Nat Methods 12:697. 10.1038/nmeth.3485

Hanahan D, and Weinberg RA. 2011. Hallmarks of cancer: the next generation. Cell 144:646674. 10.1016/j.cell.2011.02.013

Hansen TB, Jensen TI, Clausen BH, Bramsen JB, Finsen B, Damgaard CK, and Kjems J. 2013. Natural RNA circles function as efficient microRNA sponges. Nature 495:384-388. 10.1038/nature11993

Hanzelmann S, Castelo R, and Guinney J. 2013. GSVA: gene set variation analysis for microarray and RNA-seq data. BMC Bioinformatics 14:7. 10.1186/1471-2105-14-7

He L, Zhou H, Zeng Z, Yao H, Jiang W, and Qu H. 2019. Wnt/beta-catenin signaling cascade: A promising target for glioma therapy. J Cell Physiol 234:2217-2228. 10.1002/jcp.27186

Hu PS, Xia QS, Wu F, Li DK, Qi YJ, Hu Y, Wei ZZ, Li SS, Tian NY, Wei QF, Shen LJ, Yin B, Jiang T, Yuan JG, Qiang BQ, Han W, and Peng XZ. 2017. NSPc1 promotes cancer stem cell self-renewal by repressing the synthesis of all-trans retinoic acid via targeting RDH16 in malignant glioma. Oncogene 36:4706-4718. 10.1038/onc.2017.34

Kanapathipillai M. 2018. Treating p53 Mutant Aggregation-Associated Cancer. Cancers (Basel) 10. $10.3390 /$ cancers 10060154

Peer] reviewing PDF | (2021:05:61464:1:1:NEW 26 Jun 2021) 
532

533

534

535

536

537

538

539

540

541

542

543

544

545

546

547

548

549

550

551

552

553

554

555

556

557

558

559

560

561

562

563

564

565

566

567

568

569

570

571

572

573

574

575

576

577

578

579

580

581

Khan H, Patel S, and Kamal MA. 2017. Pharmacological and Toxicological Profile of Harmanebeta-Carboline Alkaloid: Friend or Foe. Curr Drug Metab 18:853-857. $10.2174 / 1389200218666170607100947$

Klaus A, and Birchmeier W. 2008. Wnt signalling and its impact on development and cancer. Nat Rev Cancer 8:387-398. 10.1038/nrc2389

Kristensen LS, Andersen MS, Stagsted LVW, Ebbesen KK, Hansen TB, and Kjems J. 2019. The biogenesis, biology and characterization of circular RNAs. Nat Rev Genet 20:675691. 10.1038/s41576-019-0158-7

Lamb J. 2007. The Connectivity Map: a new tool for biomedical research. Nat Rev Cancer 7:5460. 10.1038/nrc2044

Lamb J, Crawford ED, Peck D, Modell JW, Blat IC, Wrobel MJ, Lerner J, Brunet JP, Subramanian A, Ross KN, Reich M, Hieronymus H, Wei G, Armstrong SA, Haggarty SJ, Clemons PA, Wei R, Carr SA, Lander ES, and Golub TR. 2006. The Connectivity Map: using gene-expression signatures to connect small molecules, genes, and disease. Science 313:1929-1935. 10.1126/science.1132939

Lan YL, Zou YJ, Lou JC, Xing JS, Wang X, Zou S, Ma BB, Ding Y, and Zhang B. 2019. The sodium pump alpha1 subunit regulates bufalin sensitivity of human glioblastoma cells through the p53 signaling pathway. Cell Biol Toxicol 35:521-539. 10.1007/s10565-01909462-y

Lapointe S, Perry A, and Butowski NA. 2018. Primary brain tumours in adults. Lancet 392:432446. 10.1016/S0140-6736(18)30990-5

Lee KH, Chen CL, Lee YC, Kao TJ, Chen KY, Fang CY, Chang WC, Chiang YH, and Huang CC. 2017. Znf179 induces differentiation and growth arrest of human primary glioblastoma multiforme in a p53-dependent cell cycle pathway. Sci Rep 7:4787. 10.1038/s41598-017-05305-0

Li X, and Lee AY. 2010. Semaphorin 5A and plexin-B3 inhibit human glioma cell motility through RhoGDlalpha-mediated inactivation of Rac1 GTPase. J Biol Chem 285:32436-32445. 10.1074/jbc.M110.120451

Liang R, and Tang Y. 2020. LINC00467 knockdown repressed cell proliferation but stimulated cell apoptosis in glioblastoma via miR-339-3p/IP6K2 axis. Cancer Biomark 28:169-180. 10.3233/CBM-190939

Liu M, Wang Q, Shen J, Yang BB, and Ding X. 2019. Circbank: a comprehensive database for circRNA with standard nomenclature. RNA Biol 16:899-905. 10.1080/15476286.2019.1600395

Llaguno-Munive M, Leon-Zetina S, Vazquez-Lopez I, Ramos-Godinez MDP, Medina LA, and Garcia-Lopez P. 2020. Mifepristone as a Potential Therapy to Reduce Angiogenesis and P-Glycoprotein Associated With Glioblastoma Resistance to Temozolomide. Front Oncol 10:581814. 10.3389/fonc.2020.581814

Ma Q, Long W, Xing C, Chu J, Luo M, Wang HY, Liu Q, and Wang RF. 2018. Cancer Stem Cells and Immunosuppressive Microenvironment in Glioma. Front Immunol 9:2924. 10.3389/fimmu.2018.02924

Ma S, Meng Z, Chen R, and Guan KL. 2019. The Hippo Pathway: Biology and Pathophysiology. Annu Rev Biochem 88:577-604. 10.1146/annurev-biochem-013118-111829

Masliantsev K, Karayan-Tapon L, and Guichet PO. 2021. Hippo Signaling Pathway in Gliomas. Cells 10. 10.3390/cells10010184

Meng S, Zhou H, Feng Z, Xu Z, Tang Y, Li P, and Wu M. 2017. CircRNA: functions and properties of a novel potential biomarker for cancer. Mol Cancer 16:94. 10.1186/s12943017-0663-2

Niu X, Sun J, Meng L, Fang T, Zhang T, Jiang J, and Li H. 2020. A Five-IncRNAs SignatureDerived Risk Score Based on TCGA and CGGA for Glioblastoma: Potential Prospects

Peer) reviewing PDF | (2021:05:61464:1:1:NEW 26 Jun 2021) 
582

583

584

585

586

587

588

589

590

591

592

593

594

595

596

597

598

599

600

601

602

603

604

605

606

607

608

609

610

611

612

613

614

615

616

617

618

619

620

621

622

623

624

625

626

627

628

629

630

631

632

for Treatment Evaluation and Prognostic Prediction. Front Oncol 10:590352. 10.3389/fonc. 2020.590352

Orton RJ, Sturm OE, Vyshemirsky V, Calder M, Gilbert DR, and Kolch W. 2005. Computational modelling of the receptor-tyrosine-kinase-activated MAPK pathway. Biochem J 392:249261. 10.1042/BJ20050908

Ostrom QT, Cote DJ, Ascha M, Kruchko C, and Barnholtz-Sloan JS. 2018a. Adult Glioma Incidence and Survival by Race or Ethnicity in the United States From 2000 to 2014. JAMA Oncol 4:1254-1262. 10.1001/jamaoncol.2018.1789

Ostrom QT, Gittleman H, Truitt G, Boscia A, Kruchko C, and Barnholtz-Sloan JS. 2018b. CBTRUS Statistical Report: Primary Brain and Other Central Nervous System Tumors Diagnosed in the United States in 2011-2015. Neuro Oncol 20:iv1-iv86. 10.1093/neuonc/noy131

Pan Y, Xiao K, Li Y, Li Y, and Liu Q. 2021. RNA N6-Methyladenosine Regulator-Mediated Methylation Modifications Pattern and Immune Infiltration Features in Glioblastoma. Front Oncol 11:632934. 10.3389/fonc.2021.632934

Pinton L, Masetto E, Vettore M, Solito S, Magri S, D'Andolfi M, Del Bianco P, Lollo G, Benoit JP, Okada H, Diaz A, Della Puppa A, and Mandruzzato S. 2019. The immune suppressive microenvironment of human gliomas depends on the accumulation of bone marrowderived macrophages in the center of the lesion. J Immunother Cancer 7:58. 10.1186/s40425-019-0536-x

Quail DF, and Joyce JA. 2013. Microenvironmental regulation of tumor progression and metastasis. Nat Med 19:1423-1437. 10.1038/nm.3394

Sauvageot CM, Weatherbee JL, Kesari S, Winters SE, Barnes J, Dellagatta J, Ramakrishna NR, Stiles CD, Kung AL, Kieran MW, and Wen PY. 2009. Efficacy of the HSP90 inhibitor 17-AAG in human glioma cell lines and tumorigenic glioma stem cells. Neuro Oncol 11:109-121. 10.1215/15228517-2008-060

Saygin C, Matei D, Majeti R, Reizes O, and Lathia JD. 2019. Targeting Cancer Stemness in the Clinic: From Hype to Hope. Cell Stem Cell 24:25-40. 10.1016/j.stem.2018.11.017

Shannon P, Markiel A, Ozier O, Baliga NS, Wang JT, Ramage D, Amin N, Schwikowski B, and Ideker T. 2003. Cytoscape: a software environment for integrated models of biomolecular interaction networks. Genome Res 13:2498-2504. 10.1101/gr.1239303

Slamon DJ, Neven P, Chia S, Fasching PA, De Laurentiis M, Im SA, Petrakova K, Bianchi GV, Esteva FJ, Martin M, Nusch A, Sonke GS, De la Cruz-Merino L, Beck JT, Pivot X, Sondhi M, Wang Y, Chakravartty A, Rodriguez-Lorenc K, Taran T, and Jerusalem G. 2020. Overall Survival with Ribociclib plus Fulvestrant in Advanced Breast Cancer. $N$ Engl J Med 382:514-524. 10.1056/NEJMoa1911149

Szklarczyk D, Gable AL, Lyon D, Junge A, Wyder S, Huerta-Cepas J, Simonovic M, Doncheva NT, Morris JH, Bork P, Jensen LJ, and Mering CV. 2019. STRING v11: protein-protein association networks with increased coverage, supporting functional discovery in genome-wide experimental datasets. Nucleic Acids Res 47:D607-D613. 10.1093/nar/gky1131

Tang Z, Kang B, Li C, Chen T, and Zhang Z. 2019. GEPIA2: an enhanced web server for largescale expression profiling and interactive analysis. Nucleic Acids Res 47:W556-W560. 10.1093/nar/gkz430

Tokar T, Pastrello C, Rossos AEM, Abovsky M, Hauschild AC, Tsay M, Lu R, and Jurisica I. 2018. mirDIP 4.1-integrative database of human microRNA target predictions. Nucleic Acids Res 46:D360-D370. 10.1093/nar/gkx1144

Uhlen M, Fagerberg L, Hallstrom BM, Lindskog C, Oksvold P, Mardinoglu A, Sivertsson A, Kampf C, Sjostedt E, Asplund A, Olsson I, Edlund K, Lundberg E, Navani S, Szigyarto CA, Odeberg J, Djureinovic D, Takanen JO, Hober S, Alm T, Edqvist PH, Berling H, Tegel H, Mulder J, Rockberg J, Nilsson P, Schwenk JM, Hamsten M, von Feilitzen K, 
633

634

635

636

637

638

639

640

641

642

643

644

645

646

647

648

649

650

651

652

653

654

655

656

657

658

659

660

661

662

663

664

665

666

667

668

669

670

671

672

673

674

675

676

677

678

Forsberg M, Persson L, Johansson F, Zwahlen M, von Heijne G, Nielsen J, and Ponten F. 2015. Proteomics. Tissue-based map of the human proteome. Science 347:1260419. $10.1126 /$ science. 1260419

Vlachos IS, Zagganas K, Paraskevopoulou MD, Georgakilas G, Karagkouni D, Vergoulis T, Dalamagas T, and Hatzigeorgiou AG. 2015. DIANA-miRPath v3.0: deciphering microRNA function with experimental support. Nucleic Acids Res 43:W460-466. 10.1093/nar/gkv403

von Roemeling CA, Wang Y, Qie Y, Yuan H, Zhao H, Liu X, Yang Z, Yang M, Deng W, Bruno KA, Chan CK, Lee AS, Rosenfeld SS, Yun K, Johnson AJ, Mitchell DA, Jiang W, and Kim BYS. 2020. Therapeutic modulation of phagocytosis in glioblastoma can activate both innate and adaptive antitumour immunity. Nat Commun 11:1508. 10.1038/s41467020-15129-8

Wang H, Tao T, Yan W, Feng Y, Wang Y, Cai J, You Y, Jiang T, and Jiang C. 2015. Upregulation of miR-181s reverses mesenchymal transition by targeting KPNA4 in glioblastoma. Sci Rep 5:13072. 10.1038/srep13072

Wang R, Zhang S, Chen X, Li N, Li J, Jia R, Pan Y, and Liang H. 2018. ElF4A3-induced circular RNA MMP9 (circMMP9) acts as a sponge of miR-124 and promotes glioblastoma multiforme cell tumorigenesis. Mol Cancer 17:166. 10.1186/s12943-018-0911-0

Wesseling P, and Capper D. 2018. WHO 2016 Classification of gliomas. Neuropathol Appl Neurobiol 44:139-150. 10.1111/nan.12432

Wu P, Mo Y, Peng M, Tang T, Zhong Y, Deng X, Xiong F, Guo C, Wu X, Li Y, Li X, Li G, Zeng $Z$, and Xiong W. 2020. Emerging role of tumor-related functional peptides encoded by IncRNA and circRNA. Mol Cancer 19:22. 10.1186/s12943-020-1147-3

Xia S, Feng J, Chen K, Ma Y, Gong J, Cai F, Jin Y, Gao Y, Xia L, Chang H, Wei L, Han L, and He C. 2018. CSCD: a database for cancer-specific circular RNAs. Nucleic Acids Res 46:D925-D929. 10.1093/nar/gkx863

Yan L, and Chen YG. 2020. Circular RNAs in Immune Response and Viral Infection. Trends Biochem Sci 45:1022-1034. 10.1016/j.tibs.2020.08.006

Zhan T, Rindtorff N, and Boutros M. 2017. Wnt signaling in cancer. Oncogene 36:1461-1473. 10.1038/onc.2016.304

Zhang Q, Wang W, Zhou Q, Chen C, Yuan W, Liu J, Li X, and Sun Z. 2020. Roles of circRNAs in the tumour microenvironment. Mol Cancer 19:14. 10.1186/s12943-019-1125-9

Zhao K, Cui X, Wang Q, Fang C, Tan Y, Wang Y, Yi K, Yang C, You H, Shang R, Wang J, and Kang C. 2019. RUNX1 contributes to the mesenchymal subtype of glioblastoma in a TGFbeta pathway-dependent manner. Cell Death Dis 10:877. 10.1038/s41419-0192108-x

Zhao Z, Zhang KN, Wang Q, Li G, Zeng F, Zhang Y, Wu F, Chai R, Wang Z, Zhang C, Zhang W, Bao Z, and Jiang T. 2021. Chinese Glioma Genome Atlas (CGGA): A Comprehensive Resource with Functional Genomic Data from Chinese Gliomas. Genomics Proteomics Bioinformatics. 10.1016/j.gpb.2020.10.005

Zheng X, Chopp M, Lu Y, Buller B, and Jiang F. 2013. MiR-15b and miR-152 reduce glioma cell invasion and angiogenesis via NRP-2 and MMP-3. Cancer Lett 329:146-154. 10.1016/j.canlet.2012.10.026

Peer) reviewing PDF | (2021:05:61464:1:1:NEW 26 Jun 2021) 
679 Figure 1. Workflow diagram of the construction of a circRNA-associated ceRNA network, 680 identification and evaluation of a hub gene signature, and the prediction of potential therapeutic 681 options for glioma.

682 Figure 2. Expression profile heatmaps for 8 DECs in two GEO datasets (a, b) and basic structures 683 of the circRNAs (c). The different colors and shapes in the outer and inner ring represent the 684 different exons and the positions of MRE, RBP and ORF.

685 Figure 3. Significant signaling pathways of the 6 miRNAs utilizing the DIANA-miRPath (a), 686 chromosome distribution of DEGs from GEPIA2 (b), and the Venn graph showing the 1076 687 overlapped target genes identified with intersection of three gene sets (c).

688 Figure 4. Fnction enrichment analysis results of: Biological Process (a), Cellular Component (b), 689 Molecular Function (c), and KEGG pathways (d). The color intensity of the nodes shows the 690 degree of enrichment of this analysis. Strength is the ratio between observed counts and the 691 expected matching counts for a random list of the same size. The dot size represents the count of 692 genes in a pathway.

693 Figure 5. Forest plot of the multivariate Cox regression analysis result of LASSO significant genes.

694 Figure 6. Kaplan-Meier survival curves of risk groups in the training dataset (a) and validation 695 dataset (c), as well as the ROC curves of the hub gene signature in the training set (b) and validation 696 set (d).

697 Figure 7. A network of circRNA/miRNA/mRNA in glioma. Oval, arrow, diamond, and octagon 698 represents circRNA, miRNA, mRNA, and hub mRNAs respectively. For circRNAs, red indicates 699 upregulation and blue means downregulation. Gradual color changes of mRNAs represent 700 differences in the expression levels.

701 Figure 8. Correlation between 24 immune cells infiltration level and 46 LASSO regression analysis 702 significant genes.

703 Figure 9. The impact of candidate drugs on glioma cells. mRNA expression profiles of glioma 704 cells treated with drug(mifepristone/tretinoin) or vehicle were analyzed with GSEA. NES: 705 normalized enrichment score; FDR: false discovery rate. Negative value of NES means inhibition; 706 positive value means promotion. $\mathrm{FDR}<0.25$ were considered as significant.

707 Supp_Figure 1. No gender correlation of target mRNAs. a. PCA plot of gene expression level 708 between males and females. b. volcano plot of differentially expressed genes between males and 709 females. 
710 Supp_Figure 2. The Human Protein Atlas images of the 6 hub genes with consistent supporting 711 results. 
Figure 1

Workflow diagram of the construction of a circRNA-associated ceRNA network, identification and evaluation of a hub gene signature, and the prediction of potential therapeutic options for glioma. 


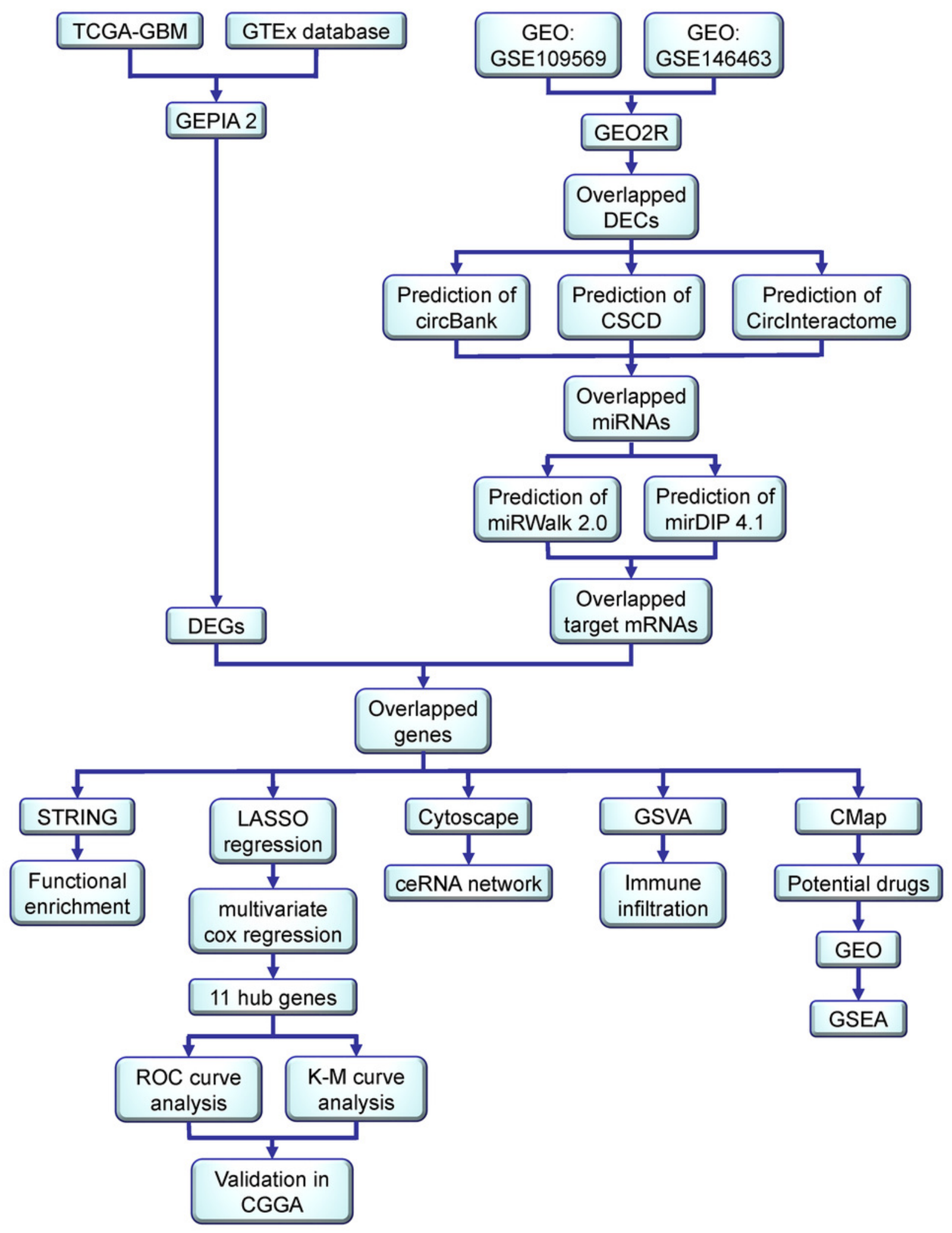


Figure 2

Expression profile heatmaps for 8 DECs in two GEO datasets $(a, b)$ and basic structures of the circRNAs $(c)$. The different colors and shapes in the outer and inner ring represent the different exons and the positions of MRE, RBP and ORF. 
A
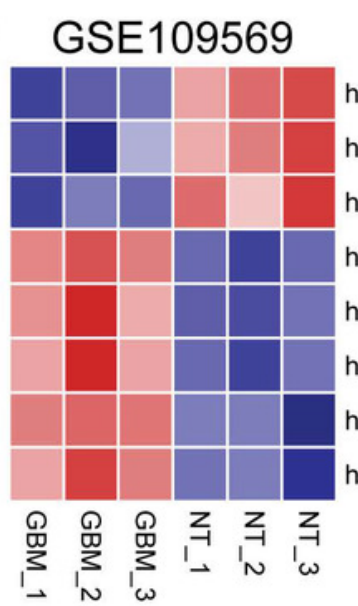

C
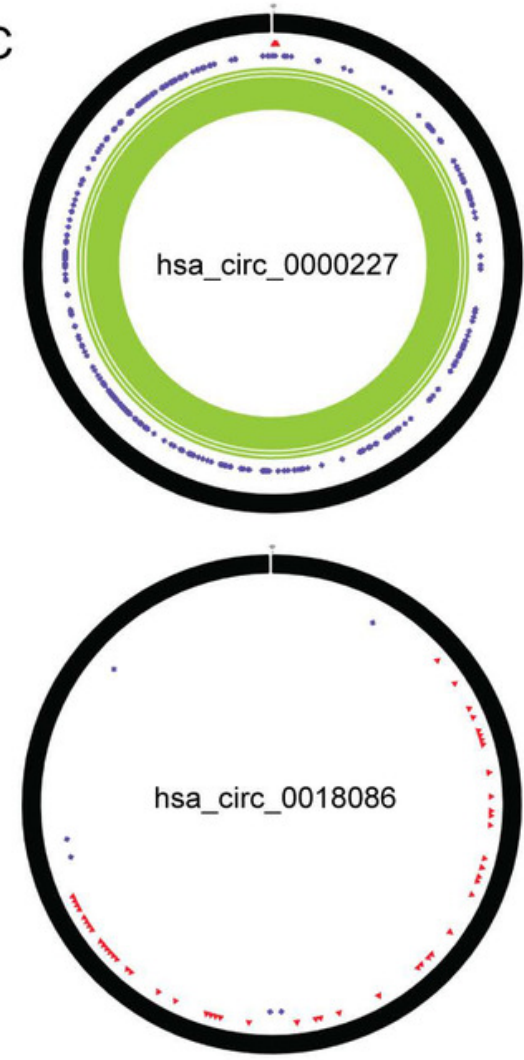

A MRE (miRNA responce element)

. RBP (RNA binding protein)

= ORF (open reading frame)
B hsa_circ_0001156
hsa_circ_0030788
hsa_circ_0034182
hsa_circ_0000227
hsa_circ_0018086
hsa_circ_0000229
hsa_circ_0036592
hsa_circ_0002765

1
0.5
0
-0.5
-1

GSE146463

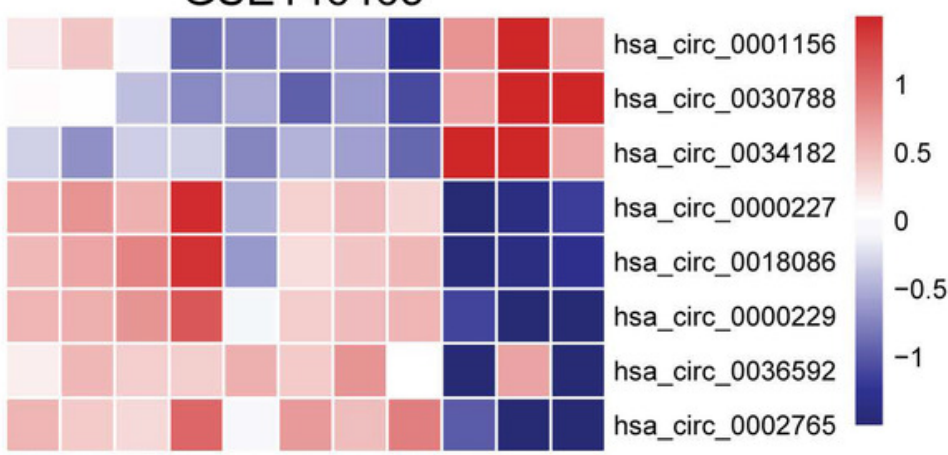

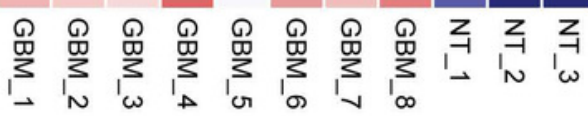
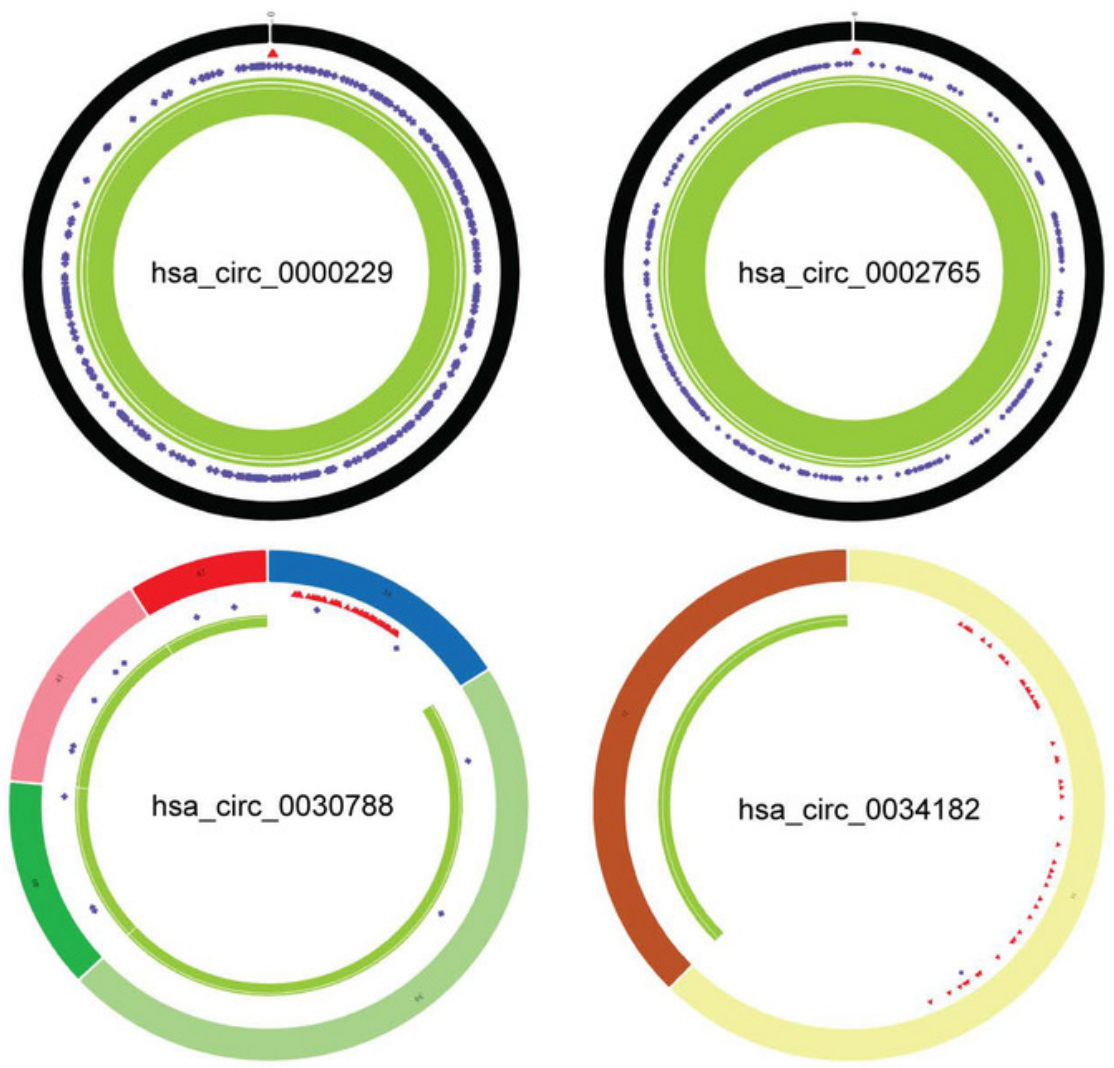
Figure 3

miRNA pathway enrichment and target genes identification.

Significant signaling pathways of the 6 miRNAs utilizing the DIANA-miRPath (a), chromosome distribution of DEGs from GEPIA2 (b), and the Venn graph showing the 1076 overlapped target genes identified with intersection of three gene sets (c). 
A

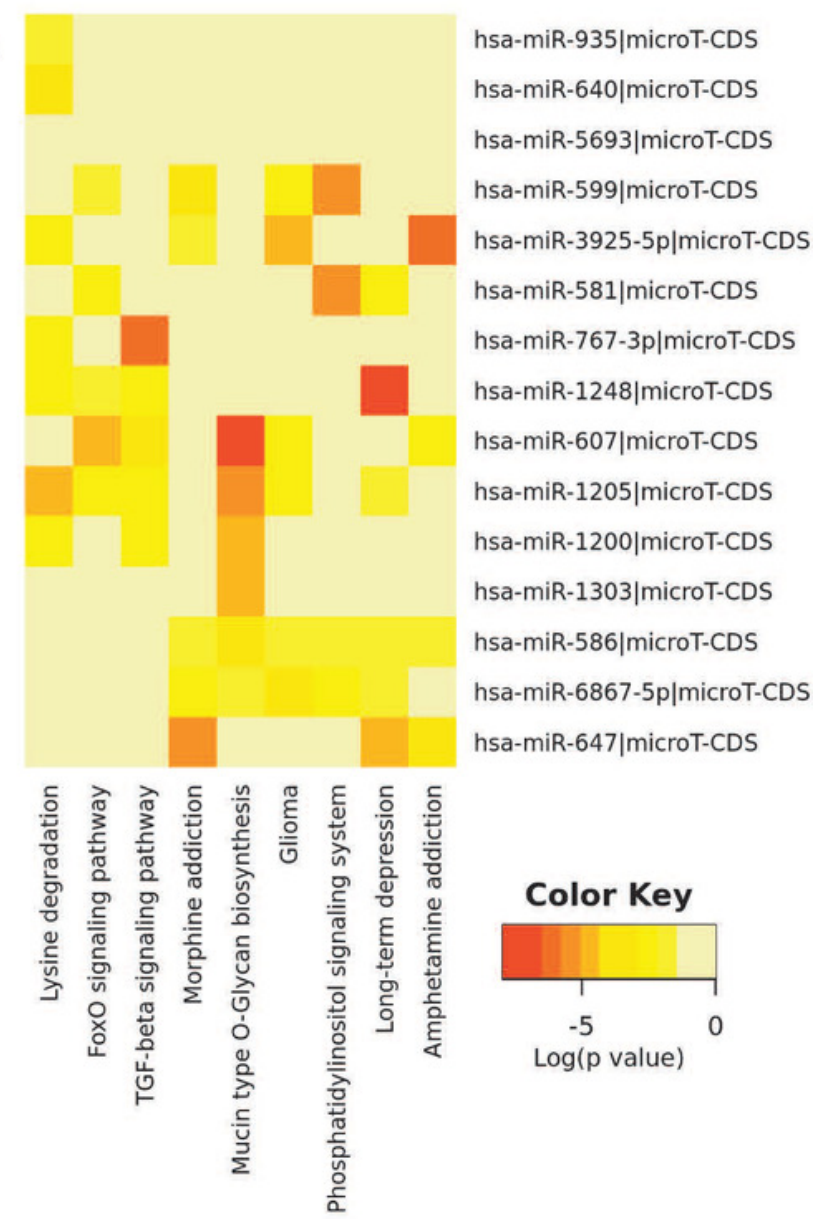

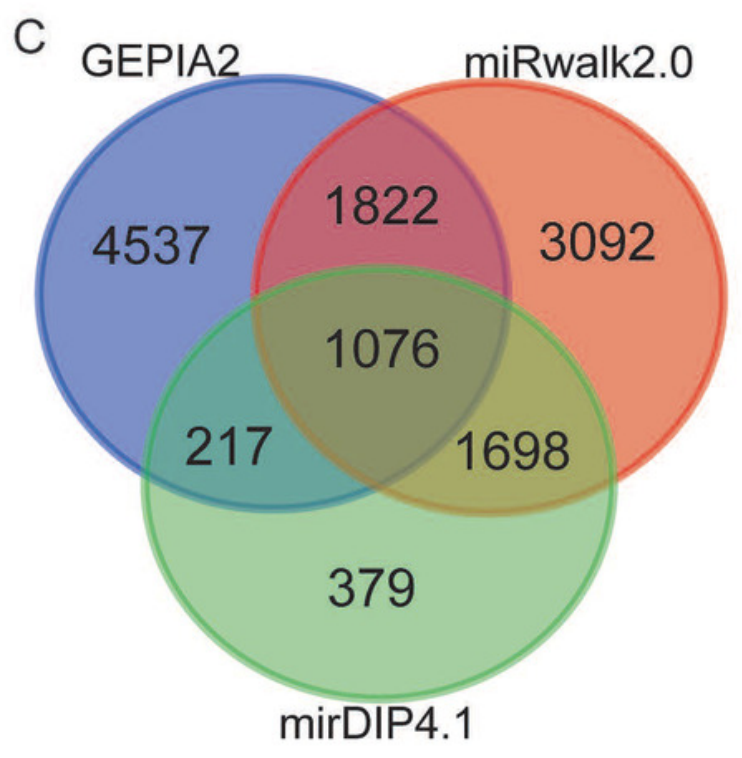

The Differentially Expressed Genes On Chromosomes

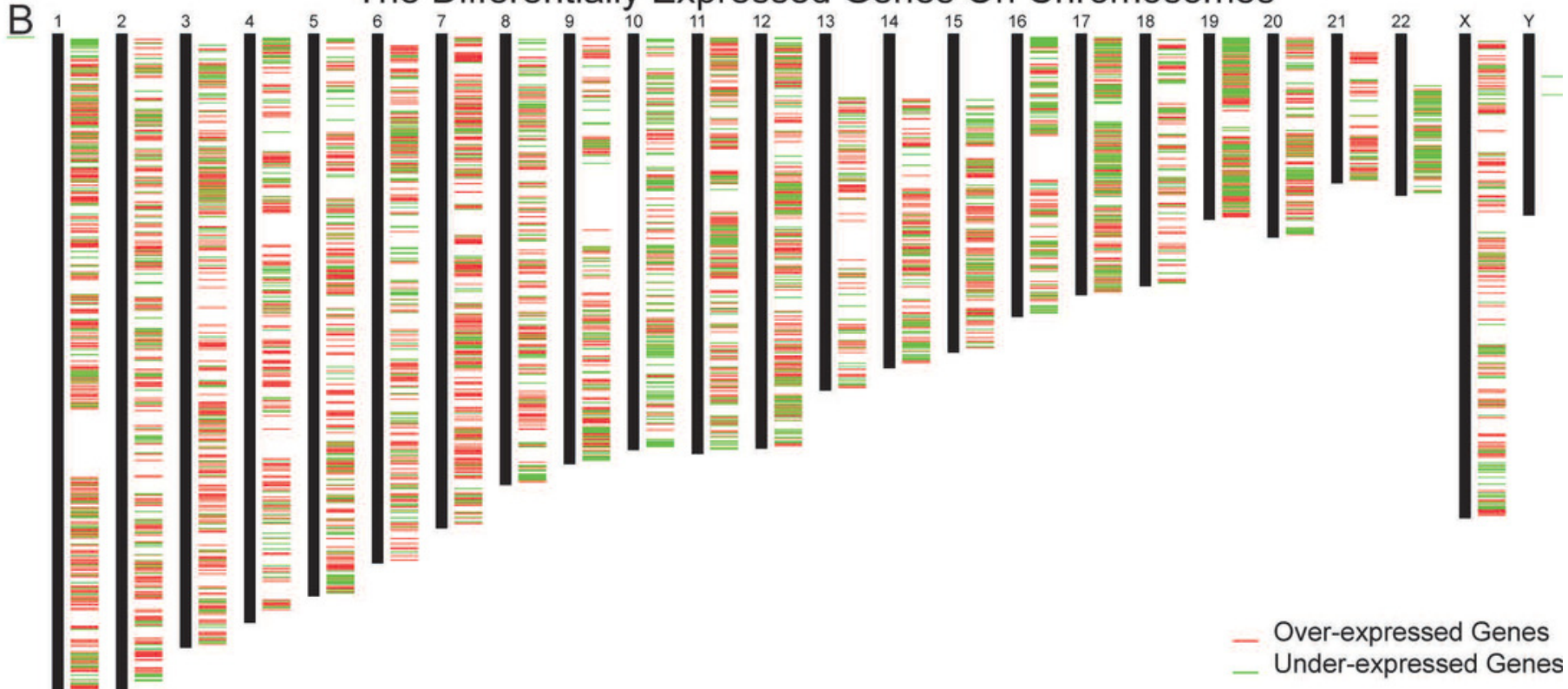


Figure 4

Function enrichment analysis results DEGs.

Biological Process (a), Cellular Component (b), Molecular Function (c), and KEGG pathways

(d). The color intensity of the nodes shows the degree of enrichment of this analysis.

Strength is the ratio between observed counts and the expected matching counts for a

random list of the same size. The dot size represents the count of genes in a pathway.

A

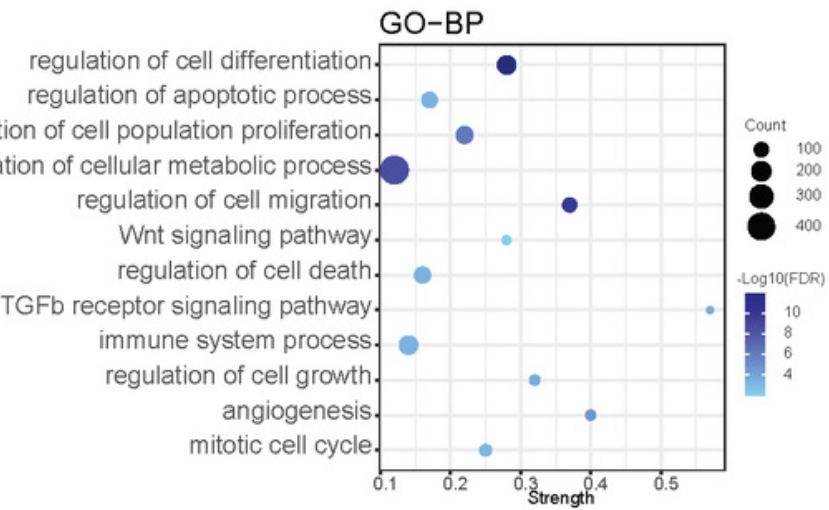

C

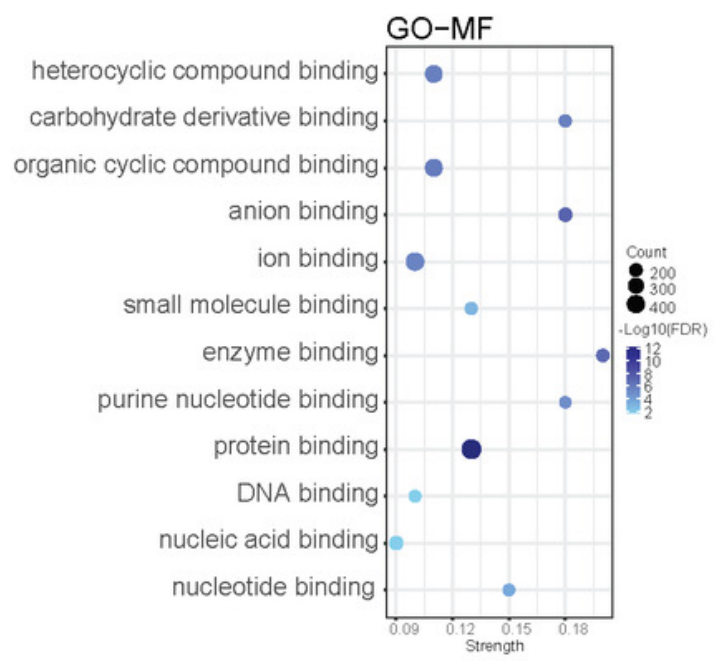

B

$\mathrm{GO}-\mathrm{CC}$

intracellular membrane-bounded organelle

intracellular organelle

membrane-bounded organelle

organelle

cell junction

cytosol

cytoplasm

cell

intracellular
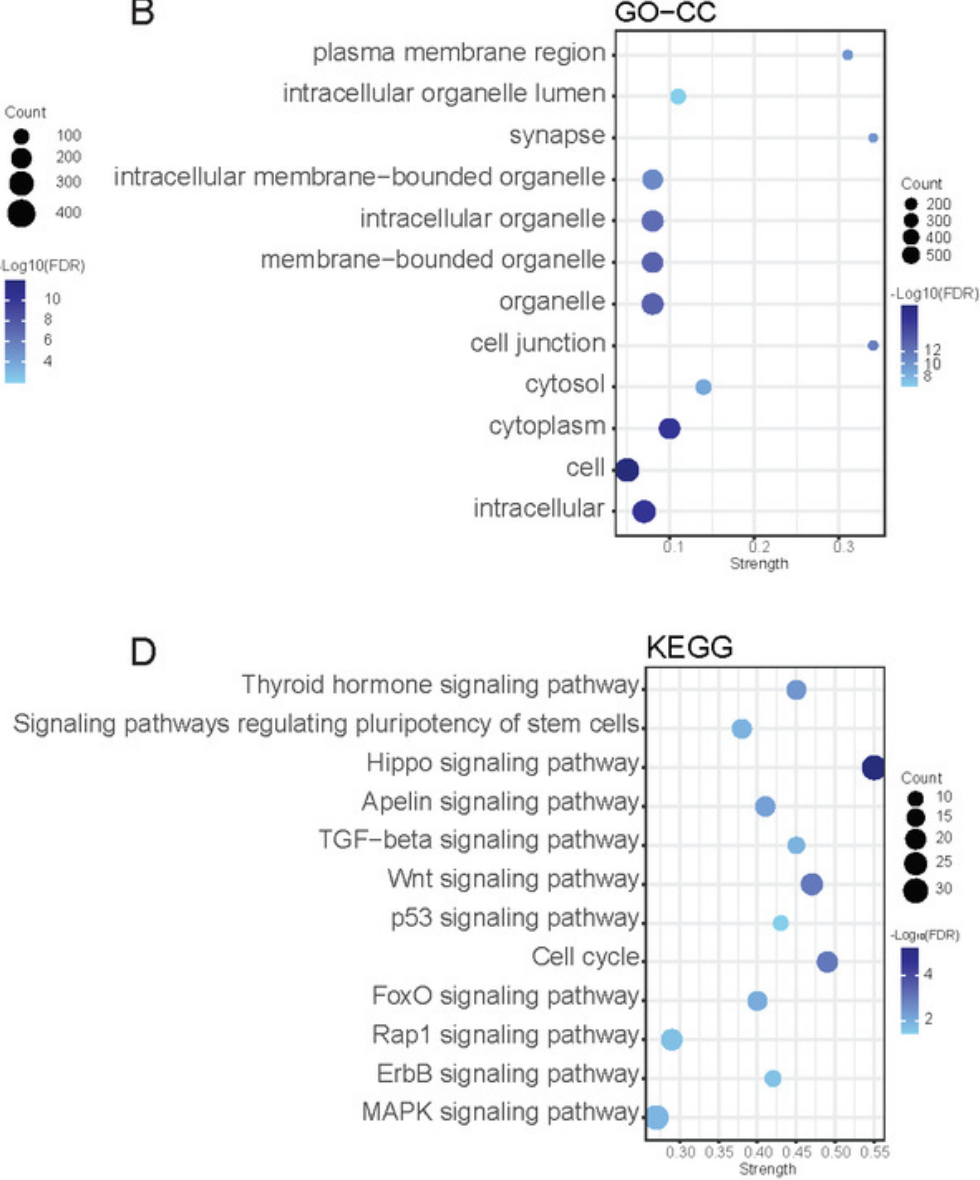
Figure 5

Forest plot of the multivariate Cox regression analysis result of LASSO significant genes.

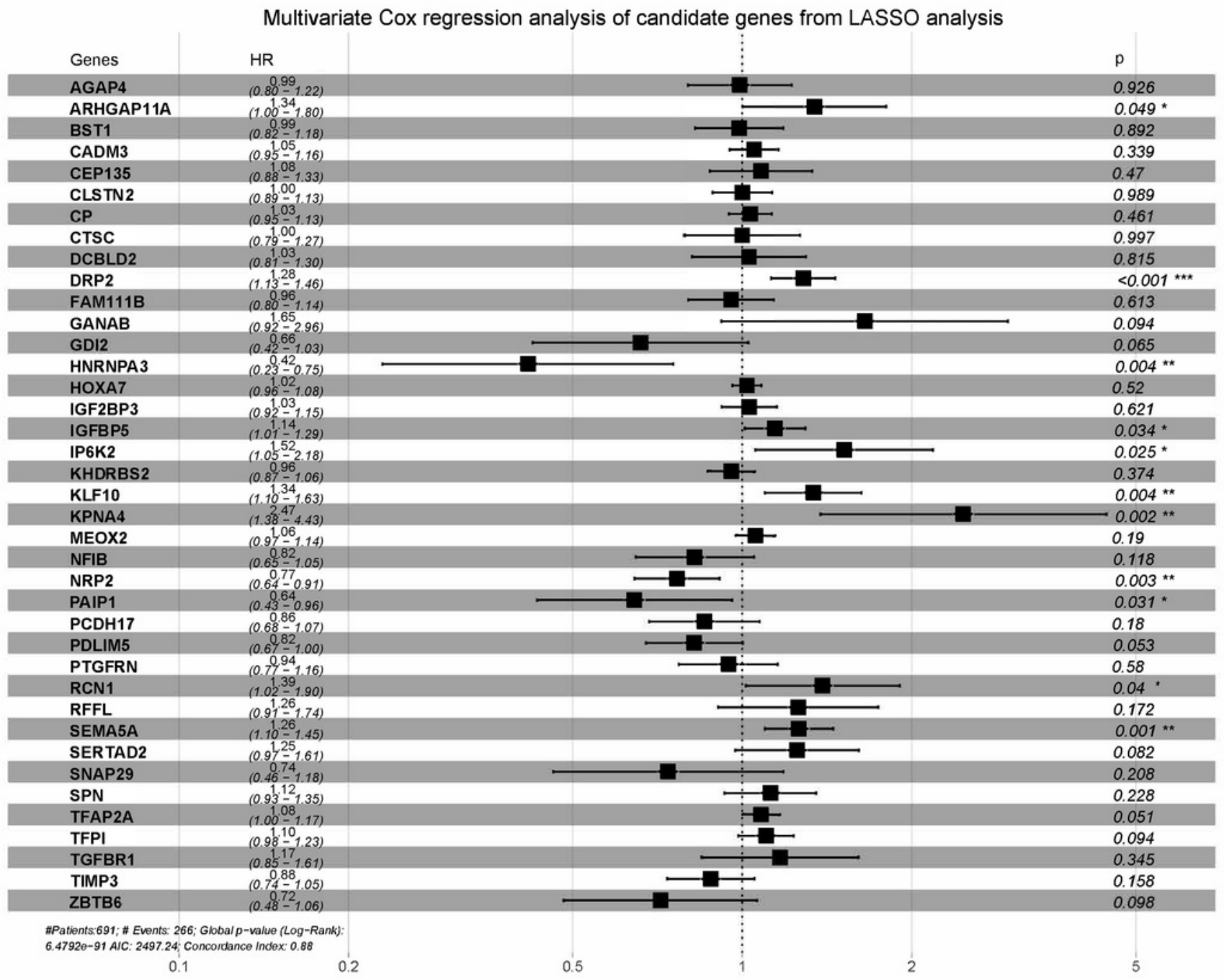


Figure 6

Kaplan-Meier survival curves analysis and ROC curves analysis

Kaplan-Meier survival curves of risk groups in the training dataset (a) and validation dataset

(c), as well as the ROC curves of the hub gene signature in the training set (b) and validation set $(d)$.

A

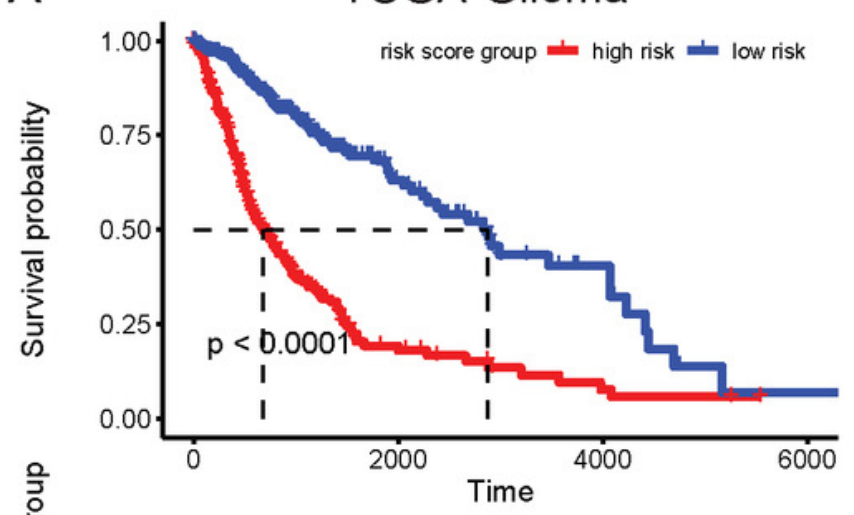

Number at risk

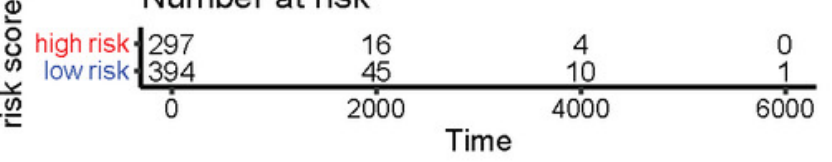

C

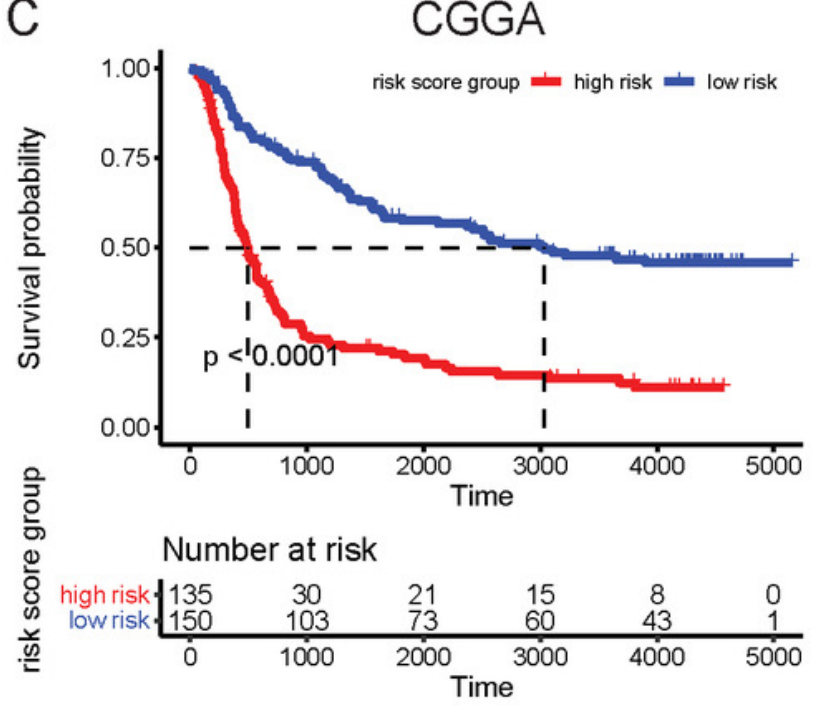

B

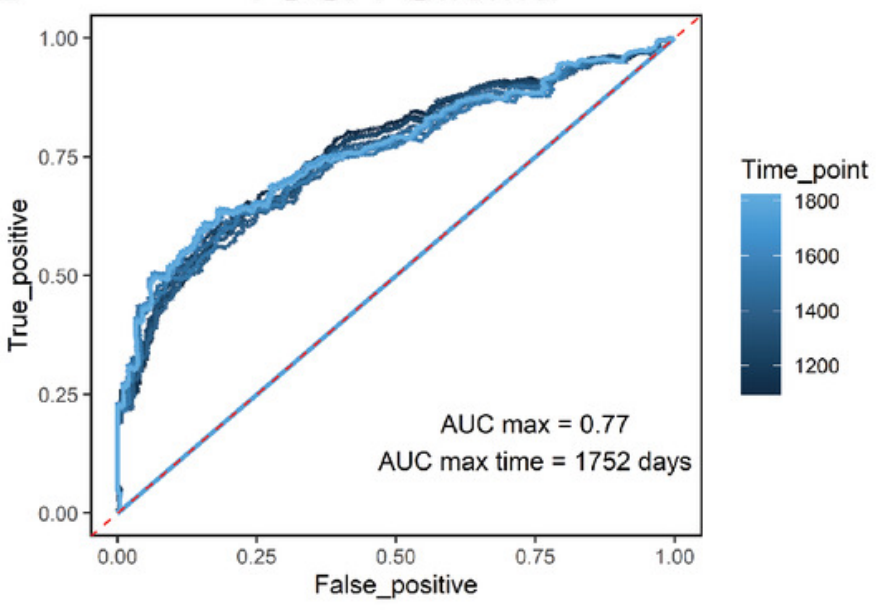

D

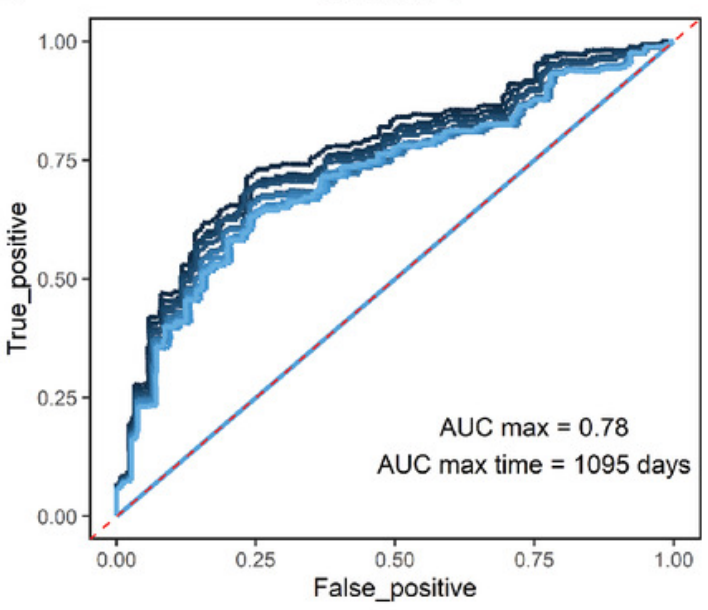

Time_point

1800 1600 1400 1200 


\section{Figure 7}

A network of circRNA/miRNA/mRNA in glioma.

Oval, arrow, diamond, and octagon represents circRNA, miRNA, mRNA, and hub mRNAs respectively. For circRNAs, red indicates upregulation and blue means downregulation. Gradual color changes of mRNAs represent differences in the expression levels.

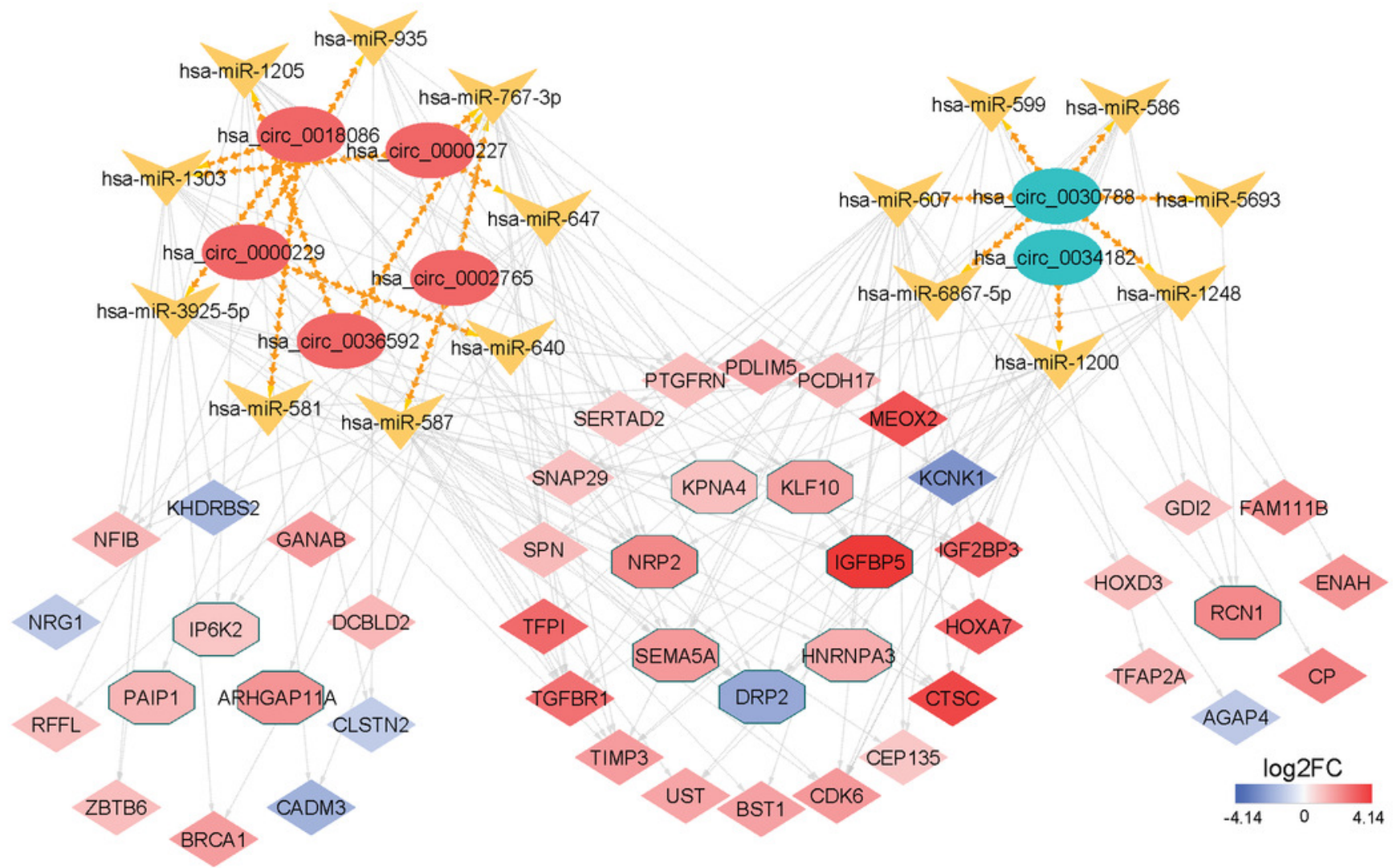


Figure 8

Correlation between 24 immune cells infiltration level and 46 LASSO regression analysis significant genes.

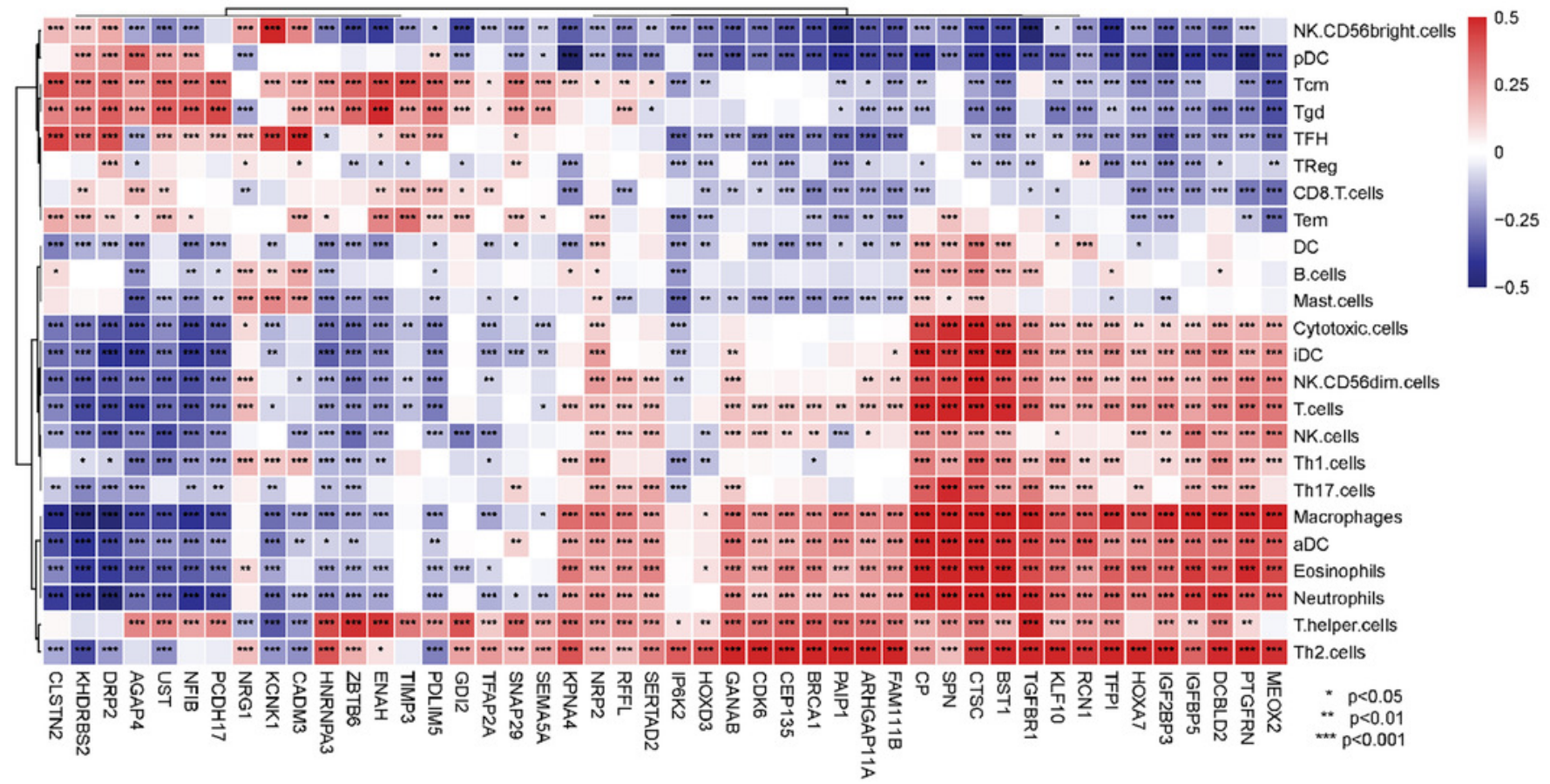




\section{Figure 9}

The impact of candidate drugs on glioma cells.

mRNA expression profiles of glioma cells treated with drug(mifepristone/tretinoin) or vehicle were analyzed with GSEA. NES: normalized enrichment score; FDR: false discovery rate. Negative value of NES means inhibition; positive value means promotion. FDR $<0.25$ were considered as significant.

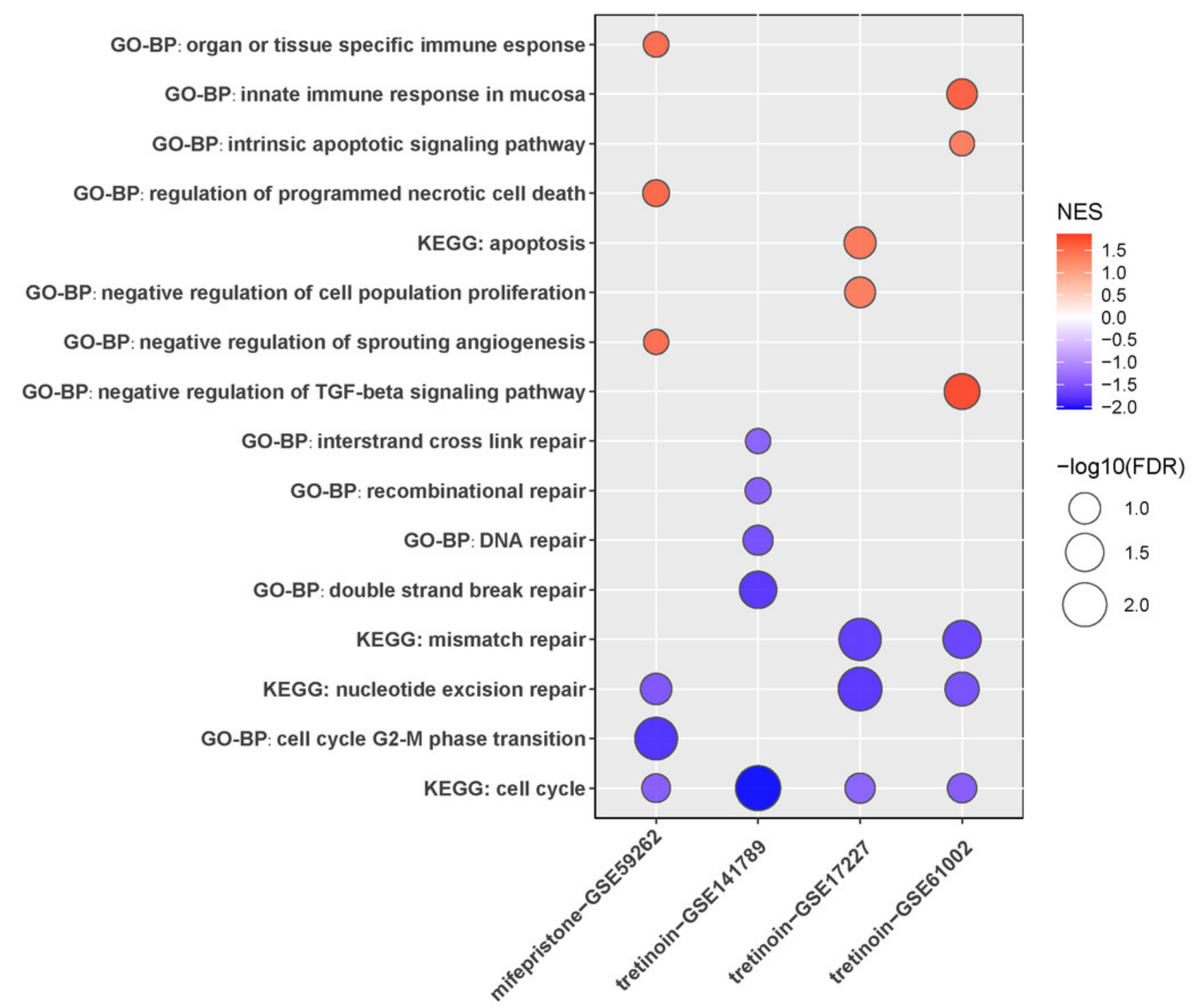


Table $\mathbf{1}$ (on next page)

Basic features of Differentially expressed circRNAs 
1 Table 1. Basic features of Differentially expressed circRNAs

\begin{tabular}{|c|c|c|c|c|c|c|c|}
\hline circBase ID & bestTranscript & Position & strand & Length & $\begin{array}{c}\text { Host gene } \\
\text { Symbol }\end{array}$ & $\begin{array}{c}\text { circRNA } \\
\text { type }\end{array}$ & Regulation \\
\hline hsa_circ_0001156 & NM_015568 & $\begin{array}{c}\text { chr20: } 37547116- \\
\text { 37547282 } \\
\text { chr13: } 101997616-\end{array}$ & + & 166 & PPP1R16B & exonic & down \\
\hline hsa_circ_0030788 & NM_052867 & $\begin{array}{c}102031004 \\
\text { chr15: } 26825465-\end{array}$ & - & 508 & NALCN & exonic & down \\
\hline hsa_circ_0034182 & NM_000814 & $\begin{array}{c}26828561 \\
\text { chr10: } 31644072-\end{array}$ & - & 221 & GABRB3 & exonic & down \\
\hline hsa_circ_0000227 & NM_030751 & $\begin{array}{c}31676195 \\
\text { chr10: } 31676052-\end{array}$ & + & 32123 & ZEB1 & intronic & up \\
\hline hsa_circ_0018086 & NM_001128128 & $\begin{array}{c}31676195 \\
\text { chr10: } 31661946-\end{array}$ & + & 143 & ZEB1 & intronic & up \\
\hline hsa_circ_0000229 & NM_030751 & $\begin{array}{c}31709678 \\
\text { chr15: } 85180577-\end{array}$ & + & 47732 & ZEB1 & intronic & up \\
\hline hsa_circ_0036592 & NR_004859 & $\begin{array}{c}85181708 \\
\text { chr10: } 31644075-\end{array}$ & + & 156 & SCAND2 & exonic & up \\
\hline hsa_circ_0002765 & NM_001128128 & 31676727 & + & 32652 & ZEB1 & intronic & up \\
\hline
\end{tabular}


Table 2 (on next page)

Table 2. Functions of miRNAs identified for ceRNA network 
1 Table 2. Functions of miRNAs identified for ceRNA network

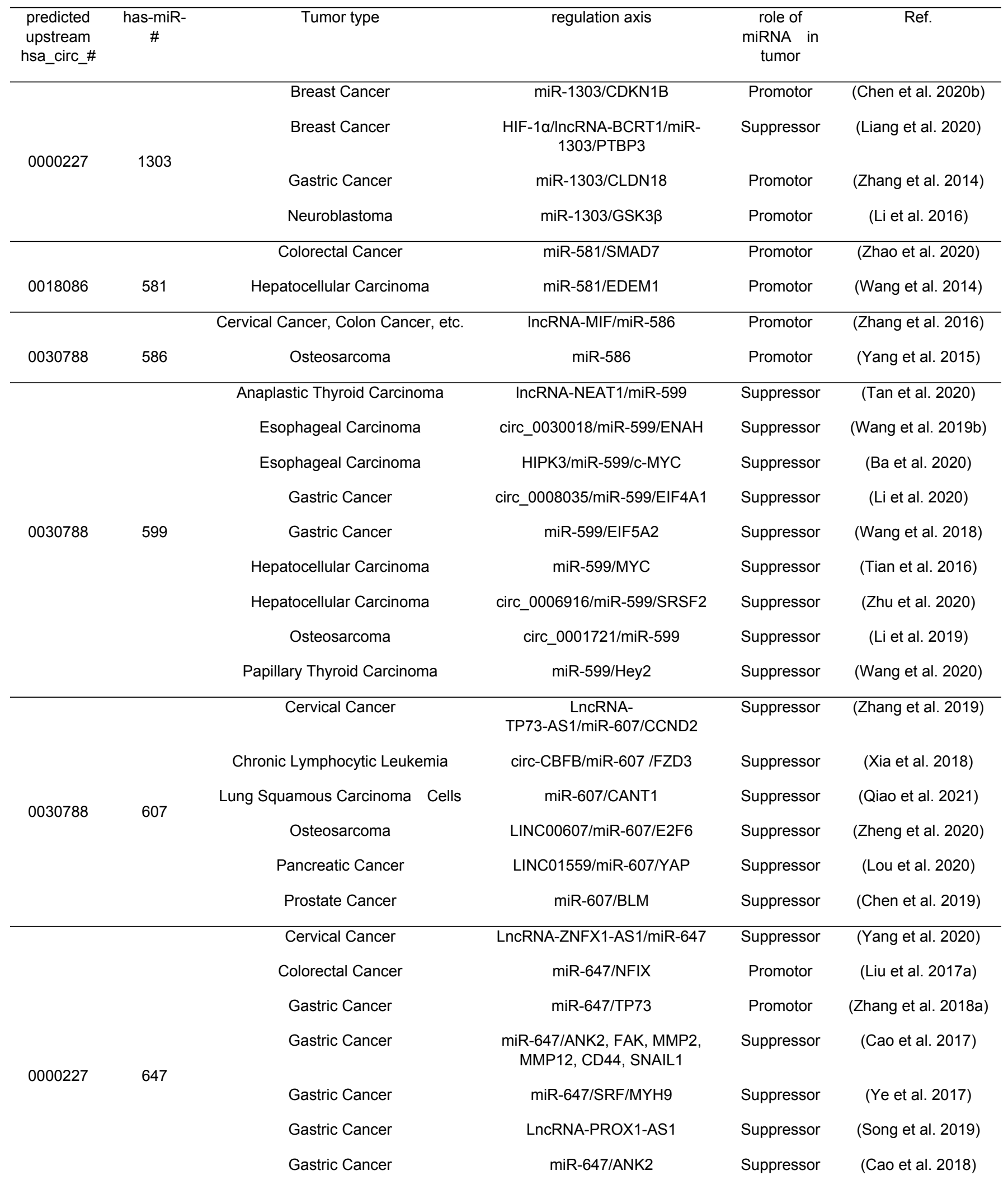




\begin{tabular}{|c|c|c|c|c|c|}
\hline \multirow{6}{*}{0000227} & \multirow{6}{*}{647} & Glioma & miR-647/HOXA9 & Suppressor & (Qin et al. 2020) \\
\hline & & Non-Small Cell Lung Cancer & $\mathrm{miR}-647 / I G F 2$ & Suppressor & (Jiang et al. 2021) \\
\hline & & Non-Small Cell Lung Cancer & miR-647/TRAF2 & Suppressor & (Zhang et al. 2018b) \\
\hline & & Osteosarcoma & circ_0001649/miR-647 & Promotor & (Sun \& Zhu 2020) \\
\hline & & Ovarian Cancer & circ-FAm53B/miR-647/MDM2 & Suppressor & (Sun et al. 2019) \\
\hline & & Prostate Cancer & $\begin{array}{c}\text { NF-KappaB/circNOLC1/miR- } \\
647 / P A Q R 4\end{array}$ & Suppressor & (Chen et al. 2020a) \\
\hline \multirow{4}{*}{$\begin{array}{l}0000227 \\
0036592\end{array}$} & \multirow{4}{*}{$767-3 p$} & Glioma & miR-767-3p & Suppressor & (Kreth et al. 2013) \\
\hline & & Hepatocellular Carcinoma & circ_0000673/miR-767-3p/SET & Suppressor & (Jiang et al. 2018) \\
\hline & & Lung Adenocarcinoma & miR-767-3p/CLDN18 & Suppressor & (Wan et al. 2018) \\
\hline & & Non-Small Cell Lung Cancer & circ_0018818/miR-767-3p & Suppressor & (Xu et al. 2020) \\
\hline \multirow{8}{*}{0000229} & \multirow{8}{*}{935} & Gastric Cancer & $\mathrm{miR}-935 / \mathrm{SOX} 7$ & Promotor & (Yang et al. 2016) \\
\hline & & Gastric Signet Ring Cell Carcinoma & miR-935/Notch1 & Suppressor & (Yan et al. 2016) \\
\hline & & Glioblastoma & miR-935/FZD6 & Suppressor & (Zhang et al. 2021) \\
\hline & & Glioma & miR-935/HIF1a & Suppressor & (Huang et al. 2020) \\
\hline & & Non-Small-Cell Lung Cancer & miR-935/SOX7 & Suppressor & (Peng et al. 2018) \\
\hline & & Non-Small-Cell Lung Cancer & $\mathrm{miR}-935 / \mathrm{E} 2 \mathrm{~F} 7$ & Suppressor & (Wang et al. 2019a) \\
\hline & & Osteosarcoma & miR-935/HMGB1 & Suppressor & (Liu et al. 2018) \\
\hline & & Liver Cancer & miR-935/SOX7 & Promotor & (Liu et al. 2017b) \\
\hline 0030788 & 1248 & Non-Small-Cell Lung Cancer & miR-1248/TYMS & Promotor & (Xu et al. 2014) \\
\hline
\end{tabular}

2

3

4 5 6

Ba Y, Liu Y, Li C, Zhu Y, and Xing W. 2020. HIPK3 Promotes Growth and Metastasis of Esophageal Squamous Cell Carcinoma via Regulation of miR-599/c-MYC Axis. Onco Targets Ther 13:19671978. 10.2147/OTT.S217087

Cao W, Wei W, Zhan Z, Xie D, Xie Y, and Xiao Q. 2017. Role of miR-647 in human gastric cancer suppression. Oncol Rep 37:1401-1411. 10.3892/or.2017.5383

Cao W, Wei W, Zhan Z, Xie D, Xie Y, and Xiao Q. 2018. Regulation of drug resistance and metastasis of gastric cancer cells via the microRNA647-ANK2 axis. Int J Mol Med 41:1958-1966. 10.3892/ijmm.2018.3381

Chen W, Cen S, Zhou X, Yang T, Wu K, Zou L, Luo J, Li C, Lv D, and Mao X. 2020a. Circular RNA CircNOLC1, Upregulated by NF-KappaB, Promotes the Progression of Prostate Cancer via miR-647/PAQR4 Axis. Front Cell Dev Biol 8:624764. 10.3389/fcell.2020.624764

Chen Y, Du M, Yusuying S, Liu W, Tan Y, and Xie P. 2020b. Nedd8-activating enzyme inhibitor MLN4924 (Pevonedistat), inhibits miR-1303 to suppress human breast cancer cell proliferation via targeting p27(Kip1). Exp Cell Res 392:112038. 10.1016/j.yexcr.2020.112038

Chen Y, Zhao J, Duan Z, Gong T, Chen W, Wang S, and Xu H. 2019. miR27b3p and miR607 cooperatively regulate BLM gene expression by directly targeting the 3'UTR in PC3 cells. Mol Med Rep 19:4819-4831. 10.3892/mmr.2019.10135 
21

Huang G, Chen J, Liu J, Zhang X, Duan H, and Fang Q. 2020. MiR-935/HIF1alpha Feedback Loop Inhibits the Proliferation and Invasiveness of Glioma. Onco Targets Ther 13:10817-10828. 10.2147/OTT.S244409

Jiang W, Wen D, Gong L, Wang Y, Liu Z, and Yin F. 2018. Circular RNA hsa_circ_0000673 promotes hepatocellular carcinoma malignance by decreasing miR-767-3p targeting SET. Biochem Biophys Res Commun 500:211-216. 10.1016/j.bbrc.2018.04.041

Jiang W, Zhao X, and Yang W. 2021. MiR-647 promotes cisplatin-induced cell apoptosis via downregulating IGF2 in non-small cell lung cancer. Minerva Med 112:312-313. 10.23736/S00264806.19.06240-2

Kreth S, Limbeck E, Hinske LC, Schutz SV, Thon N, Hoefig K, Egensperger R, and Kreth FW. 2013. In human glioblastomas transcript elongation by alternative polyadenylation and miRNA targeting is a potent mechanism of MGMT silencing. Acta Neuropathol 125:671-681. 10.1007/s00401013-1081-1

Li C, Tian Y, Liang Y, and Li Q. 2020. Circ_0008035 contributes to cell proliferation and inhibits apoptosis and ferroptosis in gastric cancer via miR-599/EIF4A1 axis. Cancer Cell Int 20:84. 10.1186/s12935020-01168-0

Li L, Guo L, Yin G, Yu G, Zhao Y, and Pan Y. 2019. Upregulation of circular RNA circ_0001721 predicts unfavorable prognosis in osteosarcoma and facilitates cell progression via sponging miR-569 and miR-599. Biomed Pharmacother 109:226-232. 10.1016/j.biopha.2018.10.072

Li Z, Xu Z, Xie Q, Gao W, Xie J, and Zhou L. 2016. miR-1303 promotes the proliferation of neuroblastoma cell SH-SY5Y by targeting GSK3beta and SFRP1. Biomed Pharmacother 83:508-513. 10.1016/j.biopha.2016.07.010

Liang Y, Song X, Li Y, Chen B, Zhao W, Wang L, Zhang H, Liu Y, Han D, Zhang N, Ma T, Wang Y, Ye F, Luo D, Li X, and Yang Q. 2020. LncRNA BCRT1 promotes breast cancer progression by targeting miR1303/PTBP3 axis. Mol Cancer 19:85. 10.1186/s12943-020-01206-5

Liu S, Qu D, Li W, He C, Li S, Wu G, Zhao Q, Shen L, Zhang J, and Zheng J. 2017a. miR647 and miR1914 promote cancer progression equivalently by downregulating nuclear factor IX in colorectal cancer. Mol Med Rep 16:8189-8199. 10.3892/mmr.2017.7675

Liu X, Li J, Yu Z, Li J, Sun R, and Kan Q. 2017b. miR-935 Promotes Liver Cancer Cell Proliferation and Migration by Targeting SOX7. Oncol Res 25:427-435. 10.3727/096504016X14747300207374

Liu Z, Li Q, Zhao X, Cui B, Zhang L, and Wang Q. 2018. MicroRNA-935 Inhibits Proliferation and Invasion of Osteosarcoma Cells by Directly Targeting High Mobility Group Box 1. Oncol Res 26:1439-1446. $10.3727 / 096504018 \times 15189093975640$

Lou C, Zhao J, Gu Y, Li Q, Tang S, Wu Y, Tang J, Zhang C, Li Z, and Zhang Y. 2020. LINC01559 accelerates pancreatic cancer cell proliferation and migration through YAP-mediated pathway. J Cell Physiol 235:3928-3938. 10.1002/jcp.29288

Peng B, Li C, Cai P, Yu L, Zhao B, and Chen G. 2018. Knockdown of miR935 increases paclitaxel sensitivity via regulation of SOX7 in nonsmallcell lung cancer. Mol Med Rep 18:3397-3402. 10.3892/mmr.2018.9330

Qiao G, Wang HB, Duan XN, and Yan XF. 2021. The effect and mechanism of miR-607/CANT1 axis in lung squamous carcinoma. Anticancer Drugs. 10.1097/CAD.0000000000001045

Qin K, Tian G, Chen G, Zhou D, and Tang K. 2020. miR-647 inhibits glioma cell proliferation, colony formation and invasion by regulating HOXA9. J Gene Med 22:e3153. 10.1002/jgm.3153

Song X, Bi Y, and Guo W. 2019. Long noncoding RNA PROX1-AS1 promotes tumor progression and aggressiveness by sponging miR-647 in gastric cancer. Minerva Med. 10.23736/S00264806.19.06223-2 
67

68

69

70

71

72

73

74

Sun D, Liu J, and Zhou L. 2019. Upregulation of circular RNA circFAM53B predicts adverse prognosis and accelerates the progression of ovarian cancer via the miR646/VAMP2 and miR647/MDM2 signaling pathways. Oncol Rep 42:2728-2737. 10.3892/or.2019.7366

Sun D, and Zhu D. 2020. Circular RNA hsa_circ_0001649 suppresses the growth of osteosarcoma cells via sponging multiple miRNAs. Cell Cycle 19:2631-2643. 10.1080/15384101.2020.1814026

Tan X, Wang P, Lou J, and Zhao J. 2020. Knockdown of IncRNA NEAT1 suppresses hypoxia-induced migration, invasion and glycolysis in anaplastic thyroid carcinoma cells through regulation of miR-206 and miR-599. Cancer Cell Int 20:132. 10.1186/s12935-020-01222-x

Tian J, Hu X, Gao W, Zhang J, Chen M, Zhang X, Ma J, and Yuan H. 2016. Identification a novel tumorsuppressive hsa-miR-599 regulates cells proliferation, migration and invasion by targeting oncogenic MYC in hepatocellular carcinoma. Am J Transl Res 8:2575-2584.

Wan YL, Dai HJ, Liu W, and Ma HT. 2018. miR-767-3p Inhibits Growth and Migration of Lung Adenocarcinoma Cells by Regulating CLDN18. Oncol Res 26:637-644. 10.3727/096504017X15112639918174

Wang C, Li S, Xu J, Niu W, and Li S. 2019a. microRNA-935 is reduced in non-small cell lung cancer tissue, is linked to poor outcome, and acts on signal transduction mediator E2F7 and the AKT pathway. Br J Biomed Sci 76:17-23. 10.1080/09674845.2018.1520066

Wang C, Tang D, Wang H, Hu G, Hu S, Li L, Min B, and Wang Y. 2019b. Circular RNA hsa_circ_0030018 acts as a sponge of miR-599 to aggravate esophageal carcinoma progression by regulating ENAH expression. J Cell Biochem. 10.1002/jcb.29507

Wang DP, Tang XZ, Liang QK, Zeng XJ, Yang JB, and XU J. 2020. microRNA-599 promotes apoptosis and represses proliferation and epithelial-mesenchymal transition of papillary thyroid carcinoma cells via downregulation of Hey2-depentent Notch signaling pathway. J Cell Physiol 235:24922505. 10.1002/jcp.29154

Wang $X$, Jin Y, Zhang H, Huang X, Zhang Y, and Zhu J. 2018. MicroRNA-599 inhibits metastasis and epithelial-mesenchymal transition via targeting EIF5A2 in gastric cancer. Biomed Pharmacother 97:473-480. 10.1016/j.biopha.2017.10.069

Wang YQ, Ren YF, Song YJ, Xue YF, Zhang XJ, Cao ST, Deng ZJ, Wu J, Chen L, Li G, Shi KQ, Chen YP, Ren H, Huang AL, and Tang KF. 2014. MicroRNA-581 promotes hepatitis B virus surface antigen expression by targeting Dicer and EDEM1. Carcinogenesis 35:2127-2133. 10.1093/carcin/bgu128

Xia L, Wu L, Bao J, Li Q, Chen X, Xia H, and Xia R. 2018. Circular RNA circ-CBFB promotes proliferation and inhibits apoptosis in chronic lymphocytic leukemia through regulating miR-607/FZD3/Wnt/betacatenin pathway. Biochem Biophys Res Commun 503:385-390. 10.1016/j.bbrc.2018.06.045

Xu J, Tian S, Yin Z, Wu S, Liu L, Qian Y, Pei D, Gao W, Xu J, Yin Y, Liu P, and Shu Y. 2014. MicroRNA-binding site SNPs in deregulated genes are associated with clinical outcome of non-small cell lung cancer. Lung Cancer 85:442-448. 10.1016/j.lungcan.2014.06.010

Xu X, Zhou X, Gao C, and Cui Y. 2020. Hsa_circ_0018818 knockdown suppresses tumorigenesis in nonsmall cell lung cancer by sponging miR-767-3p. Aging (Albany NY) 12:7774-7785. 10.18632/aging.103089

Yan C, Yu J, Kang W, Liu Y, Ma Z, and Zhou L. 2016. miR-935 suppresses gastric signet ring cell carcinoma tumorigenesis by targeting Notch1 expression. Biochem Biophys Res Commun 470:68-74. 10.1016/j.bbrc.2015.12.116

Yang L, Liu ZM, Rao YW, Cui SQ, Wang H, and Jia XJ. 2015. Downregulation of microRNA-586 Inhibits Proliferation, Invasion and Metastasis and Promotes Apoptosis in Human Osteosarcoma U2-OS Cell Line. Cytogenet Genome Res 146:268-278. 10.1159/000441073

Yang M, Cui G, Ding M, Yang W, Liu Y, Dai D, and Chen L. 2016. miR-935 promotes gastric cancer cell proliferation by targeting SOX7. Biomed Pharmacother 79:153-158. 10.1016/j.biopha.2016.01.011 
Yang Z, Ma J, Han S, Li X, Guo H, and Liu D. 2020. ZFAS1 Exerts an Oncogenic Role via Suppressing miR647 in an m(6)A-Dependent Manner in Cervical Cancer. Onco Targets Ther 13:11795-11806. 10.2147/OTT.S274492

Ye G, Huang K, Yu J, Zhao L, Zhu X, Yang Q, Li W, Jiang Y, Zhuang B, Liu H, Shen Z, Wang D, Yan L, Zhang L, Zhou H, Hu Y, Deng H, Liu H, Li G, and Qi X. 2017. MicroRNA-647 Targets SRF-MYH9 Axis to Suppress Invasion and Metastasis of Gastric Cancer. Theranostics 7:3338-3353. 10.7150/thno.20512

Zhang D, Ma S, Zhang C, Li P, Mao B, Guan X, Zhou W, Peng J, Wang X, Li S, and Jia W. 2021. MicroRNA935 Directly Targets FZD6 to Inhibit the Proliferation of Human Glioblastoma and Correlate to Glioma Malignancy and Prognosis. Front Oncol 11:566492. 10.3389/fonc.2021.566492

Zhang $\mathrm{H}$, Xue B, Wang S, Li X, and Fan T. 2019. Long noncoding RNA TP73 antisense RNA 1 facilitates the proliferation and migration of cervical cancer cells via regulating microRNA607/cyclin D2. Mol Med Rep 20:3371-3378. 10.3892/mmr.2019.10572

Zhang P, Cao L, Fan P, Mei Y, and Wu M. 2016. LncRNA-MIF, a c-Myc-activated long non-coding RNA, suppresses glycolysis by promoting Fbxw7-mediated c-Myc degradation. EMBO Rep 17:12041220. 10.15252/embr.201642067

Zhang SJ, Feng JF, Wang L, Guo W, Du YW, Ming L, and Zhao GQ. 2014. miR-1303 targets claudin-18 gene to modulate proliferation and invasion of gastric cancer cells. Dig Dis Sci 59:1754-1763. 10.1007/s10620-014-3107-5

Zhang X, Zhang M, Wang G, Tian Y, and He X. 2018a. Tumor promoter role of miR647 in gastric cancer via repression of TP73. Mol Med Rep 18:3744-3750. 10.3892/mmr.2018.9358

Zhang YS, Chen T, Cai YJ, Dong J, Bai F, Gao X, Tian L, Duan N, and Liu D. 2018b. MicroRNA-647 promotes the therapeutic effectiveness of argon-helium cryoablation and inhibits cell proliferation through targeting TRAF2 via the NF-kappaB signaling pathway in non-small cell lung cancer. Onco Targets Ther 11:6777-6784. 10.2147/OTT.S159337

Zhao X, Liu S, Yan B, Yang J, and Chen E. 2020. MiR-581/SMAD7 Axis Contributes to Colorectal Cancer Metastasis: A Bioinformatic and Experimental Validation-Based Study. Int J Mol Sci 21. 10.3390/ijms21186499

Zheng Y, Chen Z, Zhou Z, Xu X, and Yang H. 2020. Silencing of Long Non-Coding RNA LINC00607 Prevents Tumor Proliferation of Osteosarcoma by Acting as a Sponge of miR-607 to Downregulate E2F6. Front Oncol 10:584452. 10.3389/fonc.2020.584452

Zhu Z, Shen S, Zhao S, and Wang Z. 2020. Hsa_circ_0006916 Knockdown Represses the Development of Hepatocellular Carcinoma via Modulating miR-599/SRSF2 Axis. Onco Targets Ther 13:1130111313. 10.2147/OTT.S267471 


\section{Table 3(on next page)}

Table 3. Potential therapeutic options forecasted by CMap. 
1 Table 3. Potential therapeutic options forecasted by CMap.

\begin{tabular}{ccccccc}
\hline cmap name & dose & cell & score & up & down & instance_id \\
\hline fulvestrant & $10 \mathrm{nM}$ & PC3 & -1 & -0.212 & 0.661 & 4462 \\
tanespimycin & $1 \mu \mathrm{M}$ & PC3 & -0.999 & -0.407 & 0.465 & 1218 \\
mifepristone & $9 \mu \mathrm{M}$ & HL60 & -0.983 & -0.433 & 0.425 & 1569 \\
tretinoin & $1 \mu \mathrm{M}$ & PC3 & -0.955 & -0.276 & 0.558 & 1211 \\
harman & $18 \mu \mathrm{M}$ & PC3 & -0.953 & -0.495 & 0.337 & 4584 \\
miconazole & $10 \mu \mathrm{M}$ & HL60 & -0.941 & -0.453 & 0.368 & 1977 \\
ifosfamide & $15 \mu \mathrm{M}$ & PC3 & -0.933 & -0.26 & 0.554 & 5805 \\
trimethylcolchicinic acid & $12 \mu \mathrm{M}$ & PC3 & -0.931 & -0.457 & 0.356 & 4202 \\
rifampicin & $5 \mu \mathrm{M}$ & PC3 & -0.909 & -0.281 & 0.512 & 4008 \\
\hline
\end{tabular}

